\title{
APPROXIMATING CONTINUOUS FUNCTIONS BY HOLOMORPHIC AND HARMONIC FUNCTIONS
}

\author{
CHRISTOPHER J. BISHOP
}

\begin{abstract}
If $\Omega$ is a Widom domain in the plane (e.g., finitely connected) and $f$ is any bounded harmonic function on $\Omega$ which is not holomorphic, then we prove the algebra $H^{\infty}(\Omega)[f]$ contains all the uniformly continuous functions on $\Omega$. The basic tools are the solution of the $\bar{\partial}$ equation with $L^{\infty}$ estimates and some estimates on the level sets of functions in BMOA.
\end{abstract}

\section{INTRODUCTION}

Suppose $\Omega$ is an open set on the Riemann sphere, $\overline{\mathbb{C}}$, and let $H^{\infty}(\Omega)$ denote the algebra of bounded holomorphic functions on $\Omega$. If $f$ is any bounded, measurable function on $\Omega$ we let $H^{\infty}(\Omega)[f]$ denote the subalgebra of $L^{\infty}(\Omega)$ generated by $H^{\infty}(\Omega)$ and $f$. Let $C(\bar{\Omega})$ denote the uniformly continuous functions on $\Omega$ (i.e., those with continuous extension to $\bar{\Omega}$, the closure of $\Omega$ ). The purpose of this paper is to prove

Theorem 1.1. Suppose $\Omega$ is a Widom domain and that $f$ is a bounded harmonic function on $\Omega$ which is not holomorphic. Then $H^{\infty}(\Omega)[f]$ contains $C(\bar{\Omega})$.

We will not define Widom domains until $\S 3$, but should point out now that any finitely connected domain is Widom. Also note that we are not assuming $f$ has continuous boundary values, merely that it is bounded. When $\Omega$ is the unit disk, $D$, this result is due to Sheldon Axler and Allen Shields in [3]. This in turn generalized a result of Kenneth Hoffman on the unit circle, $\mathbf{T}$, which says $C(\mathbf{T}) \subset H^{\infty}(\mathbf{T})[f]$ for any $f \in L^{\infty}(\mathbf{T}) \backslash H^{\infty}(\mathbf{T})$ (see [25, p. 193 or 22, Theorem IX.1.4]). Our approach seems new even in these cases, replacing the use functional analysis with explicit constructions. The main difficulty in extending this construction to multiply connected domains is that $f$ need not have a single-valued conjugate on $\Omega$. However, if we make a strong enough assumption on $f$, then we do not need any hypothesis on $\Omega$. Here $\bar{f}$ denotes the complex conjugate of $f$.

Received by the editors May 4, 1988 .

1980 Mathematics Subject Classification (1985 Revision). 30H05, 46J15, 30E10.

Key words and phrases. Bounded holomorphic functions, function algebras, Widom domains, Wermer maximality, the Chang-Marshall theorem, the $\delta$ equation, BMOA.

The author is partially supported by a NSF Postdoctoral Fellowship. 
Theorem 1.2. Suppose $\Omega$ is an open set and that $f \in H^{\infty}(\Omega)$ is nonconstant on each component of $\Omega$. Then $C(\bar{\Omega}) \subset H^{\infty}(\Omega)[\bar{f}]$.

The same result holds if $f$ is real valued and has a single-valued conjugate on $\Omega$. Now let $A(\Omega)$ denote the holomorphic functions on $\Omega$ which have a continuous extension to $\bar{\Omega}$. It will follow from the proof of Theorem 1.2 that

Corollary 1.3. Suppose $\Omega$ is an open set and that $f \in A(\Omega)$ is nonconstant on each component of $\Omega$. Then $C(\bar{\Omega})=A(\Omega)[\bar{f}]$.

The analogous result for Theorem 1.1 is not true without some added assumptions on $\Omega$. Let $\alpha$ be continuous analytic capacity (to be defined in $\S 6$ ) and $D(x, r)$ the disk of radius $r$ centered at $x$. We will prove

Theorem 1.4. Suppose $\Omega$ is an open set and $f \in C(\bar{\Omega})$ is such that $H^{\infty}(\Omega)[f]$ contains $C(\bar{\Omega})$. If there is a $r_{0}>0$ and $\varepsilon>0$ such that

$$
\alpha(D(x, r) \backslash \Omega)>\varepsilon r
$$

for all $x \in \partial \Omega$ and $r<r_{0}$, then $A(\Omega)[f]=C(\bar{\Omega})$.

For example, suppose $\Omega$ is a Widom domain which satisfies condition (1.1) and consider $A(\Omega)$ as a function algebra on $\partial \Omega$. Suppose $f \in C(\partial \Omega) \backslash A(\Omega)$ and let $f$ also denote its harmonic extension to $\Omega$. By Theorems 1.1 and 1.4 $C(\bar{\Omega})=A(\Omega)[f]$, so restricting to the boundary we get $C(\partial \Omega)=A(\Omega)[f]$. This says $A(\Omega)$ is a maximal subalgebra of $C(\partial \Omega)$. When $\Omega$ is the unit disk, this is the Wermer maximality theorem, [51]. For general open sets, $A(\Omega)$ will not be maximal in $C(\partial \Omega)$, but in $\S 7$ we will describe all the closed subalgebras of $C(\partial \Omega)$ which contain $A(\Omega)$, at least when $\Omega$ is a union of Widom domains which satisfies (1.1).

If $K$ is a compact subset of the plane we let $K^{\circ}$ denote its interior, $C(K)$ the continuous functions on $K$ and $A(K)$ the continuous functions on $K$ which are holomorphic on $K^{\circ}$. Combining Theorems 1.1 and 1.4 as above will give

Corollary 1.5. Suppose $K$ is compact, each component of its interior is a Widom domain (e.g. finitely connected) and (1.1) holds with respect to $K^{\circ}$. If $f \in$ $C(K)$ is harmonic on $K^{\circ}$ and not holomorphic on any component of $K^{\circ}$ then $A(K)[f]=C(K)$.

Two special cases where the hypotheses of Corollary 1.5 are met are

Corollary 1.6. If $\overline{\mathbb{C}} \backslash K$ has only finitely many components and $f$ is as above, then $A(K)[f]=C(K)$.

Corollary 1.7. If $A(K)$ is a Dirichlet algebra and $f$ is as above, then $A(K)[f]=$ $C(K)$.

$A(K)$ being a Dirichlet algebra means $\operatorname{Re}(A(K))$ restricted to $\partial K$ is dense in $\operatorname{Re}(C(\partial K))$. In the proof of Corollary 1.7, we will not use this definition, but only a known geometric characterization of the sets $K$ for which this occurs. 
These corollaries generalize a result of Axler and Shields [3] where $K=\bar{D}$ is the closed unit disk. Moreover, Alexander Izzo [28] has proven that $A(K)[f]=$ $C(K)$ for every compact set $K$ and real valued $f$ as above.

Theorem 1.1 is probably true in greater generality, but we can easily produce a domain where it fails by setting $\Omega=D \backslash E$, where $E$ is a compact subset of the disk with zero analytic capacity, but such that $\Omega$ is regular for the Dirichlet problem (e.g., $E$ is a Cantor set with dimension 1/2). Now let $f \in C(\bar{\Omega})$ be the harmonic function with boundary values 1 on $\mathbf{T}=\{|z|=1\}$ and -1 on $E$. Then the level set $\{f=0\}$ contains a loop $\gamma$ and if $\left\{h_{k}\right\}$ are holomorphic on $\Omega$ then they are actually holomorphic on the interior of $\gamma$ (since $E$ is removable for bounded holomorphic functions). Thus

$$
\int_{\gamma} \sum h_{k} f^{k} d z=\int_{\gamma} h_{0} d z=0 .
$$

Hence $\int_{\gamma} h d z=0$ for every $h \in H^{\infty}(\Omega)[f]$, which certainly implies $C(\bar{\Omega}) \not \subset$ $H^{\infty}(\Omega)[f]$.

One could also consider functions $f$ which are not harmonic on $\Omega$. For example, if $f$ has one continuous derivative in a neighborhood of $\bar{D}$ then John Wermer [53] showed $A(D)[f]=C(\bar{D})$ iff the graph of $f$ in $\mathbb{C}^{2}$ is polynomially convex and $R(E)=C(E)$ where $E=\{\bar{\partial} f=0\}$ and $R(E)$ is the closure in $C(E)$ of the rational functions with poles off $E$ (also see [52, 36, 37, 40 and 41$]$ ). The graph of a harmonic function on $D$ is polynomially convex so Wermer's theorem implies Theorem 1.1 on the unit disk when $f$ is $C^{1}$. However, if we assume $f$ is $C^{1}$ on $\bar{D}$, Theorem 1.1 becomes much simpler because then $\operatorname{Re}(f) \in H^{\infty}(D)[f]$ and this easily implies $C(\bar{D}) \subset H^{\infty}(D)[f]$ (see §8). Extensions of Wermer's theorem to multiple connected domains are contained in [13 and 27].

Our strategy for proving Theorem 1.1 is as follows. Suppose we are given a bounded harmonic, but not holomorphic, function $f$ on $\Omega$ and a continuous function $g$ on $\bar{\Omega}$. Let $\Phi: D \rightarrow \Omega$ denote a uniformizing map and set $G=g$ 。 $\Phi$ and $F=f \circ \Phi$. We will then prove that $G \in H^{\infty}(D)[F]$ by constructing the required approximations. The main tools will be the solution of a $\bar{\partial}$ problem with $L^{\infty}$ estimates and some estimates on the level sets of a holomorphic function with BMO boundary values. We will then "push" this solution down to $\Omega$ by an averaging technique developed in $[11,15,16,31]$. This is the only place we use the assumption that $\Omega$ is Widom, and this restriction may be more an artifact of our approach than a real issue in the problem. Extending Theorem 1.1 further will probably require a completely different idea.

The rest of this paper is organized as follows. In $\S 2$ we will prove Theorem 1.2 since it is quite easy, and motivates the approach we will take to proving Theorem 1.1. In $\S 3$ we will discuss the uniformizing map and solve an approximation problem on the unit disk, given a certain lemma about BMOA. In $\S 4$ we will show how to deduce Theorem 1.1 from our construction on $D$ and in $\S 5$ we will prove the lemma. In $\S 6$ we will prove Theorem 1.4 and its corollaries. 
In $\S 7$ we will describe the algebras between $A(\Omega)$ and $C(\partial \Omega)$. In $\S 8$ we will discuss the Chang-Marshall theorem and sketch a proof of it using ideas from $\S \S 2$ and 3. In $\S 9$ we will discuss some special situations where Theorem 1.1 has an easier proof and we conclude with some remarks and questions in $\S 10$.

I would like to thank Sheldon Axler, Albert Baernstein, John Garnett, Don Marshall, Donald Sarason and John Wermer for helpful conversations concerning these results and Scot Adams, Alexander Izzo and the referee for their comments on the original manuscript. I am particularly grateful to Peter Jones, from whom I learned most of the techniques used here. This paper was written during my visit to the Mathematical Sciences Research Institute for the program on classical analysis, and it is a pleasure to thank MSRI and the organizers for a very pleasant and exciting year.

\section{Proof of Theorem 1.2}

Take $g \in C(\bar{\Omega})$ and suppose $f=u+i v \in H^{\infty}(\Omega)$ is nonconstant on each component of $\Omega$. We wish to prove $g \in H^{\infty}(\Omega)[\bar{f}]$. We will prove in $\S 6$ (Corollary 6.2) that we can approximate $g$ by a function which is continuous on $\bar{\Omega}$, smooth on $\Omega$ and holomorphic on $\Omega$ near $\partial \Omega$ and so we assume $g$ has this form. If we are only interested in the case when $\Omega$ is a domain we can see this by noting that if $x \in \partial \Omega$ and $r>0$ then $D(x, r) \backslash \partial \Omega$ has a component of diameter at least $r / 4$ and therefore $\gamma(D(x, r) \backslash \partial \Omega)>r / 4(\gamma$ is analytic capacity). Thus by a result of Vitushkin (e.g. [49 or 18, Theorem VIII.5.1]), $g$ can be approximated on $\partial \Omega$ by a rational function with poles off $\partial \Omega$, and therefore by a continuous function on $\bar{\Omega}$ which is holomorphic on a neighborhood of $\partial \Omega$.

We may assume $\|f\|_{\infty} \leq 1$. As usual, we set

$$
\bar{\partial}=\frac{1}{2}\left(\frac{\partial}{\partial x}+i \frac{\partial}{\partial y}\right)
$$

and recall that a function $F$ is holomorphic iff $\bar{\partial} F=0$. For each complex $\lambda$ with $|\lambda| \leq 1$,

$$
\{f=\lambda\} \cap \operatorname{supp}(\bar{\partial} g)
$$

is a finite set, so we may modify $g$ to be constant in a neighborhood of each such point. Thus we can obtain a $g_{\lambda}$ which approximates $g$ and which is holomorphic near $\partial \Omega$ and in a neighborhood of $\{f=\lambda\}$. Hence

$$
\frac{\bar{\partial} g_{\lambda}}{f-\lambda}
$$

is a smooth function of compact support. We may also assume $\infty \notin \operatorname{supp}\left(\bar{\partial} g_{\lambda}\right)$. Then

$$
h_{\lambda}(z)=\frac{1}{\pi} \int \frac{\bar{\partial} g_{\lambda}(w) d x d y}{(f(w)-\lambda)(z-w)} .
$$

is a smooth function which solves the equation $(f-\lambda) \bar{\partial} h_{\lambda}=\bar{\partial} g_{\lambda}$. Therefore $g_{\lambda}-h_{\lambda}(f-\lambda)$ is a bounded holomorphic function on $\Omega$ and approximates $g$ near the set $\{f=\lambda\}$. 
Fix $\varepsilon$ small and choose a finite collection of points $\left\{\lambda_{j}\right\}$ such that the corresponding disks $D\left(\lambda_{j}, \varepsilon /\left\|h_{\lambda_{j}}\right\|_{\infty}\right)$ cover $\bar{D}$. Set $\nu_{j}=2 \varepsilon /\left\|h_{\lambda_{j}}\right\|^{\infty}$ and take a partition of unity $\left\{P_{j}(x, y)\right\}$ on $\bar{D}$ such that $\operatorname{supp}\left(P_{j}\right) \subset D\left(\lambda_{j}, \nu\right)$. Since $f \in H^{\infty}(\Omega)$, clearly $\operatorname{Re}(f), \operatorname{Im}(f) \in H^{\infty}(\Omega)[\bar{f}]$. Define

$$
G(z)=\sum_{j}\left(g_{\lambda_{j}}(z)-h_{\lambda_{j}}(z)\left(f(z)-\lambda_{j}\right)\right) P_{j}(\operatorname{Re}(f(z)), \operatorname{Im}(f(z)))
$$

and observe $G \in H^{\infty}(\Omega)[\bar{f}]$ since the $P_{j}$ can be uniformly approximated by polynomials. To see that $G$ approximates $g$, write

$$
|g-G| \leq \sum_{j}\left|g-g_{\lambda_{j}}\right|\left|P_{j}\right|+\sum_{j}\left|h_{\lambda_{j}}\right|\left|f-\lambda_{j} \| P_{j}\right| .
$$

The first term is small since $\left|g-g_{\lambda}\right|$ was chosen small and $\sum\left|P_{j}\right|=1$. To check that the second term is small, we split it into two pieces,

$$
\sum_{\left\{j:\left|f(z)-\lambda_{j}\right| \leq 2 \nu\right\}}+\sum_{\left\{j:\left|f(z)-\lambda_{j}\right|>2 \nu\right\}} .
$$

The second sum is 0 and the first is small because

$$
\left|h_{\lambda_{j}}(z)\right|\left|f(z)-\lambda_{j}\right| \leq\left\|h_{\lambda_{j}}\right\|_{\infty} 2 \nu_{j} \leq 2 \varepsilon .
$$

This completes the proof of Theorem 1.2.

We have also proven Corollary 1.3, since it is clear that if $f$ is continuous, so is each term in the definition of $G$. We should point out that similar results have been obtained by Olin [38, §3], e.g., the case when $f$ is holomorphic on a neighborhood of $\bar{\Omega}$.

If $f$ is real valued and nonconstant on $\Omega$ and its harmonic conjugate $f^{*}$ is single valued then $\bar{F}=\exp \left(-\left(f+i f^{*}\right)\right) \exp (2 f) \in H^{\infty}(\Omega)[f]$ and the result follows from the theorem. (This was pointed out to me by Alexander Izzo, who also simplified my original proof of Theorem 1.2.) However, I do not know if the result holds for any complex-valued $f$ with single-valued harmonic conjugate.

Next we will give an application of Theorem 1.2. Suppose $\Omega$ is a bounded domain and let $L^{2}(\Omega)$ be the Hilbert space of square integrable functions with respect to area measure on $\Omega$. Let $L_{a}^{2}(\Omega)$ denote the Bergman space on $\Omega$, i.e., holomorphic functions on $\Omega$ which are in $L^{2}(\Omega)$. Let $Q$ denote the orthogonal projection from $L^{2}(\Omega)$ to $L_{a}^{2}(\Omega)$. Given a bounded function $f$ on $\Omega$ we define the Toeplitz operator $T_{f}$ by $T_{f}(h)=Q(f h)$. We let $T_{f}^{*}$ denote the adjoint of $T_{f}$ and note that if $f \in H^{\infty}(\Omega)$ then $T_{f}^{*}=T_{f}$. A pair $T_{f}, T_{g}$ is called doubly commuting if $T_{f} T_{g}^{*}=T_{g}^{*} T_{f}$. Using Theorem 1.2 we can show the following.

Corollary 2.1. Suppose $\Omega$ is a bounded domain and $f, g \in H^{\infty}(\Omega)$. Then $T_{f} T_{g}^{*}=T_{g}^{*} T_{f}$ if and only if either $f$ or $g$ is constant.

Since the proof of [2, Theorem 7], where $\Omega$ is the unit disk, goes over word for word we will not repeat it here. I would like to thank Sheldon Axler for pointing out this result to me. 
In [3, Theorem 1.2] on the unit disk is used to give a new proof of a result of Rudin [42]: that $H^{\infty}(D)+C(\bar{D})$ is a closed subalgebra of $L^{\infty}(D)$ (this generalizes Sarason's result on the unit circle [43]). In particular, it is used to show that $H^{\infty}(D)+C(\bar{D})$ is a subalgebra if it is a closed subspace. We could use Theorem 1.2 to generalize this to arbitrary open sets, but this result is already contained in the paper [14]. However, it has a completely elementary proof which does not seem to be well known, so we will give it here.

Lemma 2.2. If $\Omega$ is an open set then the closure of $H^{\infty}(\Omega)+C(\bar{\Omega})$ is a subalgebra of $L^{\infty}(\Omega)$.

Let $f \in C(\Omega)$ and $g \in H^{\infty}(\Omega)$. We need only show that $f g$ is in the closure of $H^{\infty}(\Omega)+C(\Omega)$. We may assume $\Omega$ contains $\infty$ and that $f$ is a smooth function on $\overline{\mathbb{C}}$ which is constant on a neighborhood of $\infty$. Now set $g=0$ off $\Omega$ and define

$$
\begin{aligned}
T_{f}(g)(w) & =f(w) g(w)+\frac{1}{\pi} \int \frac{g(z) \bar{\partial} f(z)}{z-w} d x d y \\
& =\frac{1}{\pi} \int \frac{(g(z)-g(w)) \bar{\partial} f(z)}{z-w} d x d y .
\end{aligned}
$$

This is Vitushkin's operator (see $\S 6$ ). Note that $T_{f}(g)$ is holomorphic on $\Omega$ since $g$ is and is bounded since $f$ is smooth. Thus

$$
f g=T_{f}(g)-\frac{1}{\pi} \int \frac{g(z) \bar{\partial} f(z)}{z-w} d x d y .
$$

The first term is in $H^{\infty}(\Omega)$ and the second term is continuous (it is a bounded function of compact support convolved with a locally integrable function). This completes the proof.

Unfortunately, it is not known when $H^{\infty}(\Omega)+C(\bar{\Omega})$ is a closed subspace of $L^{\infty}(\Omega)$. A sufficient condition can be given in terms of analytic capacity, $\gamma$, and continuous analytic capacity, $\alpha$ (defined in $\S 6$ ). It says $H^{\infty}(\Omega)+C(\bar{\Omega})$ is closed if there is an $\varepsilon>0$ such that for all $x \in \partial \Omega$ and $r<r(\Omega)$ we have

$$
\alpha(D(x, r) \backslash \Omega) \geq \varepsilon \gamma(D(x, r) \backslash \Omega) .
$$

This is proven in [14 and 20], and is conjectured to be necessary. Also see these papers for several equivalent formulations of condition (2.1).

\section{AN APPROXIMATION ON THE DISK}

Suppose $\Omega \subset \overline{\mathbb{C}}$ is a domain. If $\overline{\mathbb{C}} \backslash \Omega$ contains three or more points, then the universal covering space of $\Omega$ is the unit disk, $D$, and there is a uniformizing map $\Phi: D \rightarrow \Omega$. There is an associated group $\Gamma$ of Möbius transformations of $D$ to itself such that $\Phi \circ \gamma=\Phi$ for all $\gamma \in \Gamma$. If we let

$$
\rho(z, w)=\left|\frac{z-w}{1-\bar{w} z}\right|, \quad z, w \in D,
$$

denote the usual pseudo-hyperbolic metric on $D$, then

$$
\mathscr{F}=\{z \in D: \rho(z, 0)<\rho(z, \gamma(0)) \forall \gamma \in \Gamma\}
$$


is a fundamental domain for $\Gamma$, and is called the Dirichlet normal domain. For details see [6, 31, 32 and 39]. We let $H_{\Gamma}^{\infty}(D)$ denote the bounded holomorphic functions which are invariant under $\Gamma$ and similarly for $L_{\Gamma}^{\infty}(D)$. Observe that $H_{\Gamma}^{\infty}(D)=H^{\infty}(\Omega)$; we will use the two notations interchangably. If $\partial \Omega$ has isolated points, these are removable for bounded harmonic (and thus bounded holomorphic) functions so we may add them to $\Omega$ without changing Theorem 1.1. Thus from this point on we will assume that $\Omega$ is regular for the Dirichlet problem. This is no loss of generality, because our assumption that $\Omega$ is Widom implies it is a regular domain with at most countable many points removed [31, Lemma 2.11].

Suppose $\alpha$ is a character of $\Gamma$, i.e., a homomorphism from $\Gamma$ into the circle group, $\mathbf{T}$. We define $H_{\alpha}^{\infty}(D)$ as the bounded holomorphic functions on $D$ which satisfy

$$
f \circ \gamma=\alpha(\gamma) f
$$

for every $\gamma \in \Gamma$. Such a function is called character-automorphic. One can also think of these as bounded, multi-valued holomorphic functions on $\Omega$ which have single-valued modulus. We say $\Omega$ is a Widom domain if $H_{\alpha}^{\infty}(D)$ is nontrivial for every $\alpha$. The regular Widom domains can be characterized in terms of their Green's functions. Fix a point $w \in \Omega$ and let $G(z, w)$ be the Green's function on $\Omega$ with pole at $w$. Let $\left\{w_{j}\right\}$ denote the critical points of $G$, listed according to multiplicity. In [55] it is proven that a regular domain $\Omega$ is Widom iff

$$
\sum_{j} G\left(w_{j}, w\right)<\infty
$$

Note that any finitely connected domain satisfies this condition since the Green's function will have only finitely many critical points. We will not use Widom's result directly. Instead we will make use of the hypothesis with the following result of Pommerenke.

Fix $w=\Phi(0) \in \Omega$ and let $\left\{w_{j}\right\}$ be the critical points of the corresponding Green's function. Define $B$ to be Blaschke product on $D$ with zeros $\left\{\boldsymbol{\Phi}^{-1}(w)\right\}=\{\gamma(0): \gamma \in \Gamma\}$. Then the zeros of its derivative $B^{\prime}$ are exactly $\left\{\boldsymbol{\Phi}^{-1}\left(\left\{w_{j}\right\}\right)\right\}$. Pommerenke proves in [39] that if $\Omega$ is a Widom domain then $B^{\prime}$ is in the Nevanlinna class and its inner factor $I$ is the Blaschke product whose zeros are exactly the zeros of $B^{\prime}$. Moreover, $I$ satisfies $|I| \leq\left|B^{\prime}\right|$. In fact, these conditions characterize Widom domains. However, if we assume $\Omega$ is finitely connected, we will not need this result of Pommerenke. We can simply take $I \equiv 1$ in what follows, and use the relevant remarks in $\S 4$.

Now take $g \in C(\bar{\Omega})$ and suppose $f=u+i v$ is bounded and harmonic on $\Omega$, but not holomorphic. We wish to prove $g \in H^{\infty}(\Omega)[f]$. As is the previous section, we may assume $g$ is smooth on $\bar{\Omega}$ and is holomorphic on a neighborhood of $\partial \Omega$. Similarly, we may assume $g$ is holomorphic on a neighborhood of each critical point $w_{j}$. 
Now consider the pullbacks of $g \circ \Phi$ and $f \circ \Phi$ to $D$ which we will also denote by $g$ and $f$. They are invariant with respect to $\Gamma$ and $f=u+i v$ is harmonic, but not holomorphic on $D$. Fix $\varepsilon>0$. Our goal in this section is to construct a $G \in H^{\infty}(D)[f]$ such that $|g(z)-G(z)| \leq \varepsilon|I(z)|$.

Let $u^{*}$ and $v^{*}$ denote the harmonic conjugates of $u$ and $v$. Then

$$
h=\left(u+v^{*}\right)-i\left(v-u^{*}\right)=\bar{f}+i \bar{f}^{*}
$$

is holomorphic on $D$ and has boundary values in BMO, i.e., $h$ is in BMOA (see [22, Chapter VI] for related definitions and results). Moreover, $h$ is not constant because $f$ is not holomorphic. Thus by replacing $f$ by a scalar multiple of itself we may assume the BMO norm of $h$ is 1 .

Let $\mathscr{L}=\{2 \pi(n+i m): n, m \in \mathbb{Z}\}$ be the lattice of Gaussian integers (times $2 \pi)$. Given a function $F$ on $D$ we define

$$
E_{\lambda}=E_{\lambda}(F)=\{z \in D: F(z) \in \lambda+\mathscr{L}\}
$$

i.e., $E_{\lambda}$ is a union of level sets of $F$. To construct our approximation of $g$ we are first going to approximate $g$ on each set $E_{\lambda}(h)$ by an element of $H^{\infty}(D)$ and then "glue together" these approximations by a partition of unity constructed from $f$ and elements of $H^{\infty}(D)$. To do this we will need the following lemma:

Lemma 3.1. If $F$ is in $B M O A$ then there is a $C>0$ (depending only on the $B M O A$ norm of $F$ ) such that for any $\lambda \in \mathbb{C}$ there exists an $A \in H^{\infty}(D)$ so that for all $z \in D$

$$
C \leq \frac{|A(z)|}{\operatorname{dist}(F(z), \lambda+\mathscr{L})} \leq 1 .
$$

Note, in particular, this implies $E_{\lambda}$ is a Blaschke sequence. In fact, $E_{\lambda}(F)$ is a Blaschke sequence whenever $F$ is in the Hardy space $H^{2}(D)$ (I would like to thank Albert Baernstein for pointing this out to me). However, Lemma 3.1 itself holds only for BMOA. We will prove the lemma in $\S 5$.

Now fix $\lambda$ and let $A_{\lambda}$ be the function given by applying the lemma to $h$. We would like to find a bounded solution of $\bar{\partial} H_{\lambda}=\bar{\partial} g / I A_{\lambda}$, for then $g-$ $H_{\lambda} A_{\lambda} I$ would be an element of $H^{\infty}(D)$ which equals $g$ on $E_{\lambda}$. Unfortunately, $\bar{\partial} g / I A_{\lambda}$ may blow up near $E_{\lambda}$ so we will first replace $g$ by an approximation $G_{\lambda}$. Consider $g$ on the fundamental domain $\mathscr{F}$. Recall that $\operatorname{supp}(\bar{\partial} g) \cap \mathscr{F}$ is a compact subset of $\mathscr{F}$ and that $\bar{\partial} g$ is smooth. Since $E_{\lambda} \cap \operatorname{supp}(\bar{\partial} g)$ is a finite set we can replace $g$ by an approximation $G_{\lambda}$ which equals $g$ at each such point and which is constant in a small neighborhood of these points. Also note that $|I|$ is bounded away from zero on $\operatorname{supp}(\bar{\partial} g)$, so

$$
\frac{\left|\nabla G_{\lambda}(z)\right|\left(1-|z|^{2}\right)}{|I(z)| \operatorname{dist}(h(z), \lambda+\mathscr{L})} \leq C_{\lambda}<\infty .
$$

Since we only need to consider $\lambda \in[0,2 \pi] \times[0,2 \pi]$ we can use compactness to show there are a finite number of the $G_{\sigma}$ 's such that for any $\lambda,(3.2)$ is satisfied 
by one of these functions. Thus the constant $C_{\lambda}$ can be taken independent of $\lambda$. Moreover, we can assume $G_{\lambda}=g$ on a neighborhood of $I$ 's zeros. Arguing as before we may also assume that $\bar{\partial} g$ also vanishes on a neighborhood of $\partial \mathscr{F}$.

For each $\gamma \in \Gamma$ we make a similar modification of $g$ on $\gamma(\mathscr{F})$ using the observation that since $h$ and $h \circ \gamma$ differ only by a constant, there is a $\sigma=$ $\sigma(\gamma, \lambda)$ such that

$$
E_{\lambda} \cap \gamma(\mathscr{F})=\gamma\left(E_{\sigma} \cap \mathscr{F}\right) .
$$

Since $\bar{\partial} g=0$ near $\partial \mathscr{F}$, the modifications on adjacent copies of $\mathscr{F}$ do not interfere with each other. Thus we can build a function $G_{\lambda}$ which agrees with $g$ on $E_{\lambda}$, such that (3.2) holds for all $z \in D$, and $G_{\lambda}=g$ on a $\Gamma$-invariant neighborhood of $\{I=0\}$.

This is enough to imply that $\bar{\partial} G_{\lambda} / A_{\lambda} I$ is a Carleson measure, and so we can solve the equation with an $H_{\lambda}$ bounded on $\mathrm{T}$. We will see directly that $H_{\lambda}$ is bounded on all of $D$ by solving the $\bar{\partial}$ equation by a method implicit in Carleson's paper [10] and explicitly used by Jones in [29]. First recall that a sequence $\left\{z_{n}\right\}$ in $D$ is called interpolating with constant $M$ if for every sequence $\left\{a_{n}\right\} \in l^{\infty}$ with $\left\|\left\{a_{n}\right\}\right\|_{\infty} \leq 1$ there is a $F \in H^{\infty}(D)$ with $\|F\|_{\infty} \leq$ $M$ and $F\left(z_{n}\right)=a_{n}$ for all $n$.

If $\Omega$ is regular, as we are assuming, the inverse image under $\Phi$ of a single point is an interpolating sequence (see [31]). Moreover, since we are assuming $g$ is holomorphic near the boundary of $\Omega, \operatorname{supp}(\bar{\partial} g)$ is a compact subset of $\Omega$ and so if $x \in \operatorname{supp}(\bar{\partial} g)$ then $\Phi^{-1}(x)$ is an interpolating sequence with a uniform constant, say $M$. Let $x$ be such a point and $\left\{z_{n}\right\}$ the corresponding sequence. By [22, Theorem VII.2.1] (or [30, Theorem 6]) there are holomorphic Pehr Beurling functions $\left\{h_{n}\right\}$ which satisfy

$$
h_{n}\left(z_{n}\right)=1, \quad \sum_{n}\left|h_{n}\right| \leq C(M) .
$$

By Schwarz's lemma there is a $\delta=\delta(M)>0$ such that $h_{n}(z)>1 / 2$ if $\left|z-z_{n}\right|<\delta\left(1-\left|z_{n}\right|^{2}\right)$. Since $\operatorname{supp}(\bar{\partial} g)$ is compact we can find a finite sequence $\left\{x_{j}\right\} \subset \Omega, j=1, \ldots, N$, with corresponding sequences $\left\{z_{n}^{j}\right\}$ such that

$$
\left\{z:\left|z-z_{n}^{j}\right|<\delta\left(1-\left|z_{n}^{j}\right|^{2}\right)\right\}
$$

is a covering of $\operatorname{supp}(\bar{\partial} g)$ in $D$. Thus we can write

$$
\operatorname{supp}(\bar{\partial} g)=\bigcup D_{n}^{j}
$$

where the $D_{n}^{j}$ are disjoint sets of diameter less than $\delta\left(1-\left|z_{n}^{j}\right|^{2}\right)$. Now set

$$
H_{\lambda}(z)=\sum_{j} \sum_{n} h_{n}^{j}(z) \int_{D_{n}^{j}} \frac{\bar{\partial} G_{\lambda}(w) d x d y}{h_{n}^{j}(w) A_{\lambda}(w) I(w)(z-w)} .
$$


Then formally $\bar{\partial}\left(A_{\lambda} I H_{\lambda}\right)=A_{\lambda} I \bar{\partial} H_{\lambda}=\bar{\partial} G_{\lambda}$ so we need only check that the series converges absolutely:

$$
\begin{aligned}
\left|H_{\lambda}(z)\right| & \leq \sum_{j} \sum_{n}\left|h_{n}^{j}(z)\right| \frac{C}{1-\left|z_{n}^{j}\right|^{2}} \int_{D_{n}^{j}} \frac{d x d y}{|w-z|} \\
& \leq C \sum_{j} \sum_{n}\left|h_{n}^{j}(z)\right| \leq C \sum_{j} C(M) \leq C N C(M) .
\end{aligned}
$$

Thus $H_{\lambda}$ is a bounded holomorphic function and the bound is independent of $\lambda$, say $C_{1}$. Arguing in the same way (with $A \equiv 1$ ) we can find a bounded $H_{0}$ on $D$ such that

$$
I \bar{\partial} H_{0}=\bar{\partial} g .
$$

Now we turn to "glueing" our approximations on each $E_{\lambda}$ together. We have

$$
\left|A_{\lambda} H_{\lambda}\right| \leq C_{1} \operatorname{dist}(h, \lambda+\mathscr{L})
$$

so given $\varepsilon>0$ we can choose a, $\nu>0$ so small that $\operatorname{dist}(h(z), \lambda+\mathscr{L})<4 \nu$ implies

$$
\left|A_{\lambda}(z) H_{\lambda}(z)\right|<\varepsilon
$$

Without loss of generality we assume $2 \pi / \nu=N$ is an integer and we consider $\lambda$ 's of the form

$$
\lambda_{j k}=2 \pi(j / N+i k / N), \quad 1 \leq j, k \leq N
$$

Let $\left\{P_{j}\right\}$ be a partition on unity on $\mathbb{T}$ such that $\operatorname{supp}\left(P_{j}\right) \subset D\left(e^{i j \nu}, 2 \nu\right)$. Following the proof in [3] we observe that the function

$$
G_{t}(z)=e^{i t \operatorname{Re}(h(z))}=e^{i t\left(u(z)+v^{*}(z)\right)}=e^{i t f(z)} e^{t\left(v(z)+i v^{*}(z)\right)}
$$

is in $H^{\infty}(D)[f]$ since $e^{-t f}$ is and since $e^{-t\left(v+i v^{*}\right)}$ is a bounded holomorphic function. Similarly,

$$
F_{t}(z)=e^{i t \operatorname{Im}(h(z))}=e^{i t\left(v(z)-u^{*}(z)\right)}=e^{t f(z)} e^{-t\left(u(z)+i u^{*}(z)\right)}
$$

is in $H^{\infty}(D)[f]$. Since polynomials in $z$ and $1 / z$ are dense in $C(\mathbf{T})$ and $G_{-1}=\left(G_{1}\right)^{-1}, F_{-1}=\left(F_{1}\right)^{-1}$ we can approximate the functions

$$
P_{j}\left(G_{1}(z)\right) P_{k}\left(F_{1}(z)\right)
$$

by polynomials in $G_{1}, G_{-1}, F_{1}$ and $F_{-1}$. Since these are in $H^{\infty}(D)[f]$ we can find $Q_{j k}=\sum h_{j k l} f^{l}$ which approximate them. More precisely, for any $\eta>0$, we can find $Q_{j k}$ such that

$$
\begin{gathered}
\left|\sum_{j, k} Q_{j k}(z)-1\right|<\eta, \\
\left|\lambda_{j k}-h(z)\right| \geq 2 \nu \bmod \mathscr{L} \Rightarrow\left|Q_{j k}(z)\right| \leq \frac{2 \varepsilon}{N^{2} C_{1}},
\end{gathered}
$$




$$
\sum_{j, k}\left|Q_{j k}\right| \leq 5
$$

Now define

$$
G=\sum_{\lambda_{j k}}\left(G_{j k}-H_{j k} A_{j k} I\right) Q_{j k}+\left(g-H_{0} I\right)\left(1-\sum_{j k} Q_{j k}\right) .
$$

where $G_{j k}=G_{\lambda_{j k}}$, and similarly for $A$ and $H$. Then $G \in H^{\infty}(D)[f]$ and

$$
\begin{aligned}
|g-G| \leq & \left|\sum_{j, k}\left(G_{j k}-g\right) Q_{j k}\right|+\left|\sum_{j, k} H_{j k} A_{j k} Q_{j k}\right||I| \\
& +\left|H_{0}\right||I|\left|1-\sum_{j, k} Q_{j k}\right| .
\end{aligned}
$$

Since $H_{0}$ is bounded and $\left|1-\sum Q_{j k}\right|<\eta$, the third term is bounded by a small multiple of $|I|$ (independent of the particular choice of $\nu$ or $Q_{j k}$ ). The first term in (3.3) is identically zero in a $\Gamma$-invariant neighborhood of $\{I=0\}$ (since $G_{j k}=g$ there), and so vanishes on a set of the form $\{|I|<\delta\}$ for some small $\delta$. On the rest of $D$ it is bounded by

$$
\begin{aligned}
\left|\sum_{j, k}\left(G_{j k}-g\right) Q_{j, k}\right| & \leq \sup _{j, k}\left\|G_{j k}-g\right\| \sum_{j, k}\left|Q_{j k}\right| \\
& \leq C \sup _{j, k}\left\|G_{j k}-g\right\|
\end{aligned}
$$

which is as small as we wish. Thus the first term is also bounded by a small multiple of $|I|$. To estimate the second term note that

$$
\begin{aligned}
\left|\sum_{j, k} H_{j k} A_{j k} Q_{j k}\right| & =\sum_{\left\{j, k:\left|\lambda_{j k}-h(z)\right|<2 \nu \bmod \mathscr{L}\right\}}+\sum_{\left\{j, k:\left|\lambda_{j k}-h(z)\right| \geq 2 \nu \bmod \mathscr{L}\right\}} \\
& \leq 3 \varepsilon+N^{2} \frac{\varepsilon}{N^{2} C_{1}} C_{1} \leq 4 \varepsilon .
\end{aligned}
$$

Thus once we fix the $G_{\lambda}$ 's to make the first term in (3.3) small, this determines the $H_{\lambda}$ 's and we can then choose $\nu$ and the $Q_{j k}$ 's so that expression above is small. Therefore the second term in (3.3) is also bounded by a small multiple of $|I|$. We have now shown that for any $\varepsilon>0$ we can find a $G \in H^{\infty}(D)[f]$ such that

$$
|g(z)-G(z)| \leq \varepsilon|I(z)| \leq \varepsilon \min \left(1,\left|B^{\prime}(z)\right|\right)
$$

This completes our construction on the unit disk, except for the proof of Lemma 3.1.

Our strategy to approximate $g$ on the union of level sets

$$
E_{\lambda}=\{z: h(z) \in \lambda+2 \pi(\mathbf{Z}+i \mathbf{Z})\}
$$


might seem like an unmotivated "trick", but in fact it was essential. One can show

$$
H^{\infty}(D)[f]=H^{\infty}(D)\left[G_{t}\right]
$$

but $G_{t}$ cannot tell the difference between points $z$ and $w$ if $|\operatorname{Re}(h(z)-h(w))| \in$ $2 \pi t \mathbf{Z}$. Even if we allow a finite number of $t$ 's, say $\left\{t_{1}, \ldots, t_{n}\right\}$, the set of integers

$$
S=\left\{k:\left|e^{i t_{j} k}-e^{i t_{j}}\right|<\varepsilon, j=1, \ldots, n\right\}
$$

is infinite. In fact, it is syndetic, i.e.,

$$
\sup _{m \in \mathbf{Z}} \operatorname{dist}(m, S)<\infty
$$

(see [17, Theorem 1.21]). Thus no matter how many of the $G_{t}$ 's we allow ourselves to use, there will still be a large subset of $E_{\lambda}$ whose points cannot be well separated by these functions. Thus we must try to approximate $g$ on all of $E_{\lambda}$ by something in $H^{\infty}(D)$, which is exactly what we did.

\section{4. $\Gamma$-PROJECTION OPERATORS}

We will now use (3.4) to prove the result on $\Omega$. To do this we will use the conditional expectation operator invented by Forelli [16] (also see [11, 15 and $31])$. As in $\S 3$ let $B$ denote the Blaschke product on $D$ with zero set $\{\gamma(0): \gamma \in$ $\Gamma$ \} , $B^{\prime}$ its derivative and $I$ the inner factor of $B^{\prime}$. Then by differentiation

$$
B^{\prime}(z)=\sum_{\Gamma} \frac{\gamma^{\prime}(z)}{\gamma(z)} B(z) .
$$

Moreover, the series

$$
I(z)=\sum_{\Gamma} \frac{\gamma^{\prime}(z)}{\gamma(z)} \frac{B(z) I(z)}{B^{\prime}(z)}
$$

converges absolutely on $\mathscr{F}$ (see $[39, \S 4])$. Now define

$$
E(f)=\sum_{\Gamma}(f \circ \gamma(z)) \frac{\gamma^{\prime}(z) B(z)}{\gamma(z) B^{\prime}(z)} .
$$

One easily sees that if $f$ is invariant under $\Gamma$, then $E(f)=f$. Also, if $f \in H^{\infty}(D)$ then $E(f)$ is meromorphic on $D$, with poles possibly occurring at the zeros of $B^{\prime}$. Applying $E$ to the functions $g$ and $G$ constructed in $\S 3$ and using (3.4)

$$
\begin{aligned}
|g-E(G)| & =|E(g-G)(z)| \\
& \leq \sum_{\Gamma}\left|\frac{(g-G) \circ \gamma(z)}{I(z)}\right|\left|\frac{\gamma^{\prime}(z)}{\gamma(z)} \frac{B(z) I(z)}{B^{\prime}(z)}\right| \\
& \leq \varepsilon \sum_{\Gamma}\left|\frac{\gamma^{\prime}(z)}{\gamma(z)}\right|\left|\frac{B(z) I(z)}{B^{\prime}(z)}\right| \\
& \leq C \varepsilon .
\end{aligned}
$$


Thus $E(G)$ uniformly approximates $g$. Finally, we need that

$$
E(G) \in H^{\infty}(\Omega)[f] .
$$

We can write $G$ as $g-H_{0} I$ plus a finite sum of terms which look like

$$
F q f^{l}=\left(G_{\lambda}-H_{\lambda} A_{\lambda} I-\left(g-H_{0} I\right)\right) q f^{l}
$$

where $q \in H^{\infty}(D)$ and $q f^{l}$ is a term that comes from expanding one of the $Q_{j k}$ into powers of $f$ times elements of $H^{\infty}(D)$. Applying $E$ to this expression we get

$$
E(F q) f^{l}
$$

since $f^{l}$ is $\Gamma$-invariant. Because $F q$ is holomorphic, $E(F q)$ is meromorphic and invariant. But we also have

$$
|F q| \leq\left(\left|G_{\lambda}-g\right|+\left|H_{\lambda} A_{\lambda} I\right|+\left|H_{0} I\right|\right)|q| \leq C|I|
$$

so just as above, $E(F q)$ is bounded, and thus holomorphic. A similar, but simpler, argument shows $E\left(g-H_{0} I\right)$ is bounded and holomorphic on $\Omega$. Thus $E(G) \in H^{\infty}(\Omega)[f]$ and it approximates $g$, completing the proof of Theorem 1.1 .

If $\Omega$ were finitely connected there is a simpler method we could have used to deduce the approximation on $\Omega$ from the one on $D$. This would have been to use

Theorem 4.1 (Forelli [16]). If $\Omega$ is a regular, finitely connected plane domain then there is a bounded linear operator $P: L^{\infty}(D) \rightarrow L_{\Gamma}^{\infty}(D)$ such that

(1) $P\left(H^{\infty}(D)\right) \subset H_{\Gamma}^{\infty}(D)$.

(2) If $F \in L_{\Gamma}^{\infty}(D)$ and $G \in L^{\infty}(D)$ then $P(F G)=F P(G)$.

(3) $P(1)=1$.

This is also proven in [11]. To see how this proves Theorem 1.1, suppose $g \in C(\bar{\Omega})$ and $f$ is bounded and harmonic on $\Omega$ but not holomorphic. Fix $\varepsilon>0$ and suppose there are bounded holomorphic functions $\left\{h_{k}\right\}$ on $D$ such that

$$
\left\|g \circ \Phi-\sum_{k} h_{k}(f \circ \Phi)^{k}\right\|_{\infty}<\varepsilon .
$$

Then using the theorem,

$$
\left\|g-\sum_{k} P\left(h_{k}\right) f^{k}\right\|_{\infty}<\|P\| \varepsilon
$$

and $\left\{P\left(h_{k}\right)\right\}$ are bounded holomorphic functions on $\Omega$. With this approach, the construction on $D$ could have been simpler, for we could drop all references to $B^{\prime}$ and $I$ in $\S 3$. These projections also exist for some infinitely connected domains, e.g., the homogeneous Denjoy domains described in $\S 10$ (see [11]). However, any such domain is necessarily Widom, and there do exist Widom 
domains for which Theorem 4.1 is unknown, so the extra work we have done is not completely unjustified. (Note that if Theorem 4.1 holds for a domain $\Omega$, then the corona theorem is true on $\Omega$. The corona problem is still open for general Widom domains, indeed, a proof for this case would imply it for arbitrary planar domains.)

\section{Proof of Lemma 3.1}

Lemma 3.1 is just a way of quantifying the fact that an analytic function $F$ in BMO cannot pass near $\mathscr{L}$ very often. The John-Nirenberg theorem implies $F$ cannot visit points of $\mathscr{L}$ that are far away too often, and the analyticity of $F$ says it cannot remain in a neighborhood of any one point too long. This is very reminiscent of the geometric charaterizations of BMO functions and domains found in [25, 44 and 45], and in fact Lemma 3.1 characterizes BMOA. Its converse is essentially contained in Theorem 3 of [4], which gives a "value distribution" characterization of BMOA. To prove Lemma 3.1 we will use the following estimates. Here $P_{z}$ denotes the Poisson kernel on $\mathrm{T}$ with respect to the point $z$,

$$
P_{z}\left(e^{i \theta}\right)=\frac{1}{2 \pi} \frac{1-|z|^{2}}{\left|e^{i \theta}-z\right|^{2}} .
$$

As before, let $\mathscr{L}=\{2 \pi(n+i m): n, m \in \mathbb{Z}\}$ and set

$$
\varphi(z)=\log (\operatorname{dist}(z, \mathscr{L})) .
$$

Then $\varphi$ is superharmonic on $\mathbb{C} \backslash \mathscr{L}$, since it is the infimum of harmonic functions, and is bounded above by $\log (\sqrt{2} \pi)$.

Lemma 5.1. Suppose $F \in B M O A$ with norm 1 . For $z \in D$ let $\lambda=\lambda(z)$ be a closest point of $\mathscr{L}$ to $F(z)$. Let $X=X(\lambda)=\left\{e^{i \theta}:\left|F\left(e^{i \theta}\right)-\lambda\right| \leq 1\right\}$. Then we have

$$
\begin{gathered}
\int_{\mathbf{T} \backslash X} \log \left|F\left(e^{i \theta}\right)-\lambda\right| d \theta \leq C_{1}<\infty . \\
\int_{\mathbf{T} \backslash X} \varphi \circ F\left(e^{i \theta}\right) P_{z}\left(e^{i \theta}\right) d \theta \geq-C_{2}>-\infty . \\
\int_{\mathbf{T}} \varphi \circ F\left(e^{i \theta}\right) P_{z}\left(e^{i \theta}\right) d \theta \geq \log |F(0)-\lambda|-C_{3} .
\end{gathered}
$$

The constants $C_{1}, C_{2}, C_{3}$ are independent of $F$ and $\lambda$.

Without loss of generality we may assume $z=0$. By the John-Nirenberg theorem (e.g., [22, Theorem VI.2.1]), and the fact that $|F-\lambda|-\sqrt{2} \pi \leq$ $|F-F(0)| \leq|F-\lambda|+\sqrt{2} \pi$, 


$$
\begin{aligned}
\int_{\mathbf{T} \backslash X} \log |F-\lambda| d \theta & =\sum_{k=1}^{\infty} \int_{\{\theta: k \leq|F-\lambda|<k+1\}} \log |F-\lambda| d \theta \\
& \leq \sum_{k=0}^{\infty} \int_{\{\theta: k \leq|F-F(0)|<k+10\}} \log ^{+}|F-\lambda| d \theta \\
& \leq \sum_{k=0}^{\infty} \log (k+10)|\{\theta:|F-F(0)| \geq k\}| \\
& \leq C \sum_{k=0}^{\infty} \log (k) e^{-C k} \\
& \leq C_{1} .
\end{aligned}
$$

This proves the first claim. To prove the second, define

$$
X_{\sigma}^{\delta}=\left\{\theta:\left|F\left(e^{i \theta}\right)-\sigma\right|<\delta\right\} .
$$

Clearly the collection $\left\{X_{\sigma}^{\delta}\right\}, \sigma \in \mathscr{L}$, is disjoint for $\delta \leq \pi$. By the JohnNirenberg theorem there is an absolute $\delta>0$ such that $|F(z)-\sigma|<\delta$ implies

$$
\left|\left\{\theta \in I_{z}:\left|F\left(e^{i \theta}\right)-\sigma\right|>1\right\}\right|<\frac{1}{2}\left|I_{z}\right|
$$

where $I_{z}$ is the interval of length $2(1-|z|)$ centered at $z /|z|$. In terms of $X_{\sigma}=X_{\sigma}^{1}$, this means

$$
\frac{\left|X_{\sigma} \cap I_{z}\right|}{\left|I_{z}\right|} \geq \frac{1}{2}
$$

Now consider the usual decomposition of $D$ into dyadic Carleson boxes. Let $\left\{Q_{j}^{\sigma}\right\}$ denote the disjoint collection of boxes which are maximal with respect to the property that there is a point $z_{j}$ in the top half of $Q$ such that $\left|F\left(z_{j}\right)-\sigma\right|=$ $\delta$. By Fatou's theorem $\left\{I_{j}^{\sigma}\right\}$, the bases of these cubes, cover almost every point of $X_{\sigma}^{\delta}$. Suppose $z$ is such a point and let $I$ be the base of the corresponding box. Since $\log |F-\sigma|$ is subharmonic,

$$
\begin{aligned}
\log (\delta) & =\log |F(z)-\sigma| \\
& \leq \int_{\mathbf{T}} \log \left|F\left(e^{i \theta}\right)-\sigma\right| P_{z}\left(e^{i \theta}\right) d \theta \\
& =\int_{I}+\int_{(\mathbf{T} \backslash I) \cap X_{\sigma}^{!}}+\int_{(\mathbf{T} \backslash I) \backslash X_{\sigma}^{!}}
\end{aligned}
$$

The second term is negative and by the first part of the lemma the third term is less than a constant, so

$$
\log (\delta) \leq \int_{I} \log \left|F\left(e^{i \theta}\right)-\sigma\right| P_{z}\left(e^{i \theta}\right) d \theta+C_{1}
$$

which implies

$$
-C \leq C \log \delta-C \leq \frac{1}{|I|} \int_{I} \log |F(z)-\sigma| d \theta .
$$


Let

$$
Y=X_{\lambda} \cup \bigcup_{\sigma \in \mathscr{L}, \sigma \neq \lambda} X_{\sigma}^{\delta}
$$

Then,

$$
\begin{aligned}
\int_{\mathbf{T} \backslash X} \varphi \circ F\left(e^{i \theta}\right) d \theta & =\int_{\mathbf{T} \backslash Y} \varphi \circ F\left(e^{i \theta}\right) d \theta+\sum_{\sigma \in \mathscr{L}, \sigma \neq \lambda} \int_{X_{\sigma}^{\delta}} \varphi \circ F\left(e^{i \theta}\right) d \theta \\
& \geq C \log (\delta)+\sum_{\sigma \neq \lambda} \int_{X_{\sigma}^{\delta}} \log |F-\lambda| \\
& \geq-C+\sum_{\sigma \neq \lambda} \sum_{j} \int_{I_{j}^{\sigma}} \log |F-\lambda| \\
& \geq-C+\sum_{\sigma \neq \lambda} \sum_{j} C\left|I_{j}^{\sigma}\right| \log \delta \\
& \geq-C-C \sum_{\sigma \neq \lambda} \sum_{j}\left|I_{j}^{\sigma}\right| \\
& \geq-C-C \sum_{\sigma}\left|X_{\sigma}^{1}\right| \\
& \geq-C_{2}
\end{aligned}
$$

as required to prove the second claim. To prove the last part of the lemma, note that using parts (1) and (2),

$$
\begin{aligned}
\int_{\mathbf{T}} \varphi \circ F d \theta & =\int_{X} \varphi \circ F d \theta+\int_{\mathbf{T} \backslash X} \varphi \circ F d \theta \\
& \geq \int_{X} \varphi \circ F d \theta-C_{2} \\
& =\int_{X} \log |F-\lambda| d \theta-C_{2} \\
& \geq \int_{\mathbf{T}} \log |F-\lambda| d \theta-C_{1}-C_{2} \\
& \geq \log |F(0)-\lambda|-C_{3}
\end{aligned}
$$

where the last step is the subharmonicity of $|F-\lambda|$. This completes the proof of Lemma 5.1.

We now turn to the proof of Lemma 3.1. Since we may assume $F$ has BMO norm $1,\|F-\alpha\|_{\infty} \geq 1$ for every constant $\alpha$. Thus by composing with a Möbius transformation we may assume $\operatorname{dist}(F(0), \lambda+\mathscr{L}) \geq 1 / 2$. By considering $F(r z)$ and taking limits we may also assume $F$ is analytic on $\bar{D}$. With $\varphi$ as before, note that $\varphi \circ F$ is superharmonic on $D$ except at the points where $F(z) \in$ $\lambda+\mathscr{L}$. Let $B$ denote the Blaschke product which vanishes at exactly these points (according to multiplicity) and note that $\varphi \circ F-\log |B|$ is superharmonic 
on $D$, and by [22, Theorem I.6.7] will have a harmonic minorant $S$ iff

$$
\begin{aligned}
\inf _{0<r<1} \int_{\mathbf{T}} \varphi \circ F\left(r e^{i \theta}\right)-\log \left|B\left(e^{i \theta}\right)\right| d \theta & =\int_{\mathbf{T}} \varphi \circ F\left(e^{i \theta}\right)-\log \left|B\left(e^{i \theta}\right)\right| d \theta \\
& =\int_{\mathbf{T}} \varphi \circ F\left(e^{i \theta}\right) d \theta \\
& \geq-\infty .
\end{aligned}
$$

However, this is exactly part (3) of Lemma 5.1 , so $S$ exists. It is bounded above because it is harmonic on $D$ and satisfies

$$
S \leq \varphi \circ F-\log |B| \leq \varphi \circ F \leq \log \sqrt{2} \pi .
$$

on $\mathbf{T}$. Thus,

$$
A(z)=B(z) e^{S(z)+i S^{*}(z)}
$$

is a bounded holomorphic function which satisfies

$$
|A| \leq e^{\varphi \circ F} \leq \operatorname{dist}(F, \mathscr{L}) .
$$

To get the other inequality we first write $B$ as a product

$$
B=\prod_{\sigma \in \mathscr{L}} B_{\sigma}
$$

where each $B_{\sigma}$ is the Blaschke product corresponding to the zero set $\{F=\sigma\}$. Corollary 6.1 of [4] says that

$$
\log \left|B_{\sigma}(z)\right| \geq-C e^{-c|\sigma-F(z)|} .
$$

(In the notation of that paper, $N(\lambda, z, F)=-\log \left|B_{\lambda}(z)\right|$.) Thus if $\lambda$ is the closest lattice point to $F(z)$,

$$
\sum_{\sigma \neq \lambda} \log \left|B_{\sigma}(z)\right|>-C_{4}>-\infty
$$

Now suppose $z \in D$ and let $\lambda$ be the closest lattice point to $F(z)$. Using Lemma 5.1, the facts that $\log \left|B_{\lambda}\right|=0$ on $T$ and that $\log |F-\lambda|-\log \left|B_{\lambda}\right|$ is harmonic on $D$,

$$
\begin{aligned}
S(z) & =\int_{\mathbf{T}} \varphi \circ F P_{z} d \theta \\
& =\int_{X_{\lambda}} \log |F-\lambda| P_{z} d \theta+\int_{\mathbf{T} \backslash X_{\lambda}} \varphi \circ F P_{z} d \theta \\
& \geq \int_{X_{\lambda}} \log |F-\lambda| P_{z} d \theta-C_{2} \\
& \geq \int_{\mathbf{T}} \log |F-\lambda| P_{z} d \theta-C_{2}-C_{1} \\
& =\int_{\mathbf{T}}\left(\log |F-\lambda|-\log \left|B_{\lambda}\right|\right) P_{z} d \theta-C_{5} \\
& =\log |F(z)-\lambda|-\log \left|B_{\lambda}(z)\right|-C_{5} \\
& =\varphi \circ F(z)-\log \left|B_{\lambda}(z)\right|-C_{5} .
\end{aligned}
$$


Thus we have shown

$$
\log |A|=\log |B|+|S| \geq \varphi \circ F-C_{4}-C_{5}
$$

which implies

$$
|A| \geq e^{-C_{4}-C_{5}} \operatorname{dist}(F, \mathscr{L})
$$

as desired. This completes the proof of Lemma 3.1 .

\section{Proof of TheOREM 1.4}

We now turn to the proof of Theorem 1.4. The techniques are not new, but we will review the details for completeness. We start by recalling the definition of continuous analytic capacity, $\alpha$. If $E$ is a compact subset of the plane, let $A_{E}$ denote the continuous functions on the Riemann sphere which are holomorphic off $E$. Then

$$
\alpha(E)=\sup \left\{\left|f^{\prime}(\infty)\right|: f \in A_{E},\|f\|_{\infty} \leq 1, f(\infty)=0\right\} .
$$

The regular analytic capacity $\gamma$ is defined similarly by taking the supremum over $f \in H^{\infty}(\overline{\mathbb{C}} \backslash E)$. For details and the basic properties of $\alpha$ and $\gamma$ see [21]. We will also need to recall Vitushkin's localization operator ([49], but our presentation follows [19]),

$$
T_{\varphi}(f)(w)=\varphi(w) f(w)+\frac{1}{\pi} \int \frac{f(z) \bar{\partial} \varphi}{z-w} d x d y=\frac{1}{\pi} \int \frac{(f(z)-f(w)) \bar{\partial} \varphi}{z-w} d x d y
$$

where $\varphi$ is smooth and has compact support. $T_{\varphi}$ has the following properties:

(1) If $X$ is the support of $\varphi$,

$$
\left\|T_{\varphi}(f)\right\|_{\infty} \leq 2 \operatorname{diam}(X)\|\bar{\partial} \varphi\|_{\infty} \sup _{w, z \in X}|f(z)-f(w)| .
$$

(2) $T_{\varphi}(f)$ is continuous where $f$ is.

(3) $T_{\varphi}(f)$ is analytic off $X$.

(4) $T_{\varphi}(f)$ is analytic where $f$ is.

(5) $f-T_{\varphi}(f)$ is analytic on the interior of $\varphi^{-1}(1)$.

Now suppose we have $g \in C(\bar{\Omega}), f \in C(\bar{\Omega})$ and $\left\{h_{k}\right\} \subset H^{\infty}(\Omega)$ such that

$$
\left\|g-\sum_{k=0}^{N} h_{k} f^{k}\right\|_{\infty}<\varepsilon
$$

We assume the $h_{k}$ 's have a common bound $M$. We will use Vitushkin's operator to find functions $\left\{\tilde{h}_{k}\right\} \subset A(\Omega)$ such that

$$
\left\|g-\sum_{k=0}^{N} \tilde{h}_{k} f^{k}\right\|_{\infty} \leq C \varepsilon
$$

To simplify notation we consider

$$
\vec{h}=\left(h_{0}, h_{1}, h_{2}, \ldots, h_{\Lambda^{*}}\right), \quad \vec{f}=\left(1, f, f^{2}, \ldots, f^{N}\right)
$$


as $\mathbb{C}^{N+1}$ valued functions, and note that the inequality above now has the form

$$
\|\vec{h} \cdot \vec{f}-g\|_{\infty}<\varepsilon .
$$

Fix $\eta>0$ (to be chosen later), and let $\delta$ be so small that $|z-w|<\delta$ implies $|g(z)-g(w)|<\eta$ and $\left|f^{k}(z)-f^{k}(w)\right|<\eta$ for all $k=1, \ldots, N$. If we fix a $z \in \bar{\Omega}$ and take $w \in D(z, \delta) \cap \Omega$, then

$$
\begin{aligned}
|\vec{h}(w) \cdot \vec{f}(z)-g(z)| \leq & |\vec{h}(w) \cdot \vec{f}(w)-g(w)| \\
& +|\vec{h}(z) \cdot(\vec{f}(z)-\vec{f}(w))|+|g(z)-g(w)| \\
\leq & \varepsilon+M \eta+\eta \leq 2 \varepsilon
\end{aligned}
$$

if $\eta$ is small enough. Now let $\left\{\varphi_{k}\right\}$ be a collection of smooth "bump functions" such that

(1) $\operatorname{supp}\left(\varphi_{j}\right) \subset D\left(x_{j}, \delta\right)$ for some $x_{j} \in \partial \Omega$.

(2) $\left|\nabla \varphi_{j}\right| \leq C \delta^{-1}$.

(3) $\varphi_{j}(z)=1$ for all $z \in D\left(x_{j}, \delta / 2\right)$.

(4) If $\Phi=\sum \varphi_{j}$, then $\partial \Omega \subset \Phi^{-1}(1)$.

Since $\alpha(D(x, \delta / 4) \backslash \Omega)>\nu \delta$ for some $\nu>0$, there is (as in [19]) a function $a_{j}$ which is continuous on all of $\overline{\mathbb{C}}$, holomorphic on $\Omega \cup\left(\overline{\mathbb{C}} \backslash D\left(x_{j}, \delta / 4\right)\right)$, and satisfies

$$
\begin{aligned}
& \left|a_{j}(z)\right| \geq C \nu, \quad z \in \operatorname{supp}\left(\bar{\partial} \varphi_{j}\right) . \\
& \left|a_{j}(z)\right| \leq \frac{C}{1+\left|z-x_{j}\right|^{3}}, \quad z \in \mathbb{C} .
\end{aligned}
$$

For each $j$ choose a point $z_{j} \in \Omega \cap D\left(x_{j}, \delta / 4\right)$ and set $\vec{H}_{j}=\vec{h}\left(z_{j}\right)$. Now define

$$
\begin{gathered}
\vec{A}_{j}(w)=\vec{h}(w) \varphi_{j}(w)+\frac{a_{j}(w)}{\pi} \int \frac{\vec{h}(z) \bar{\partial} \varphi_{j}(z)}{a_{j}(z)(z-w)} d x d y \\
\vec{B}_{j}(w)=\vec{H}_{j} \varphi_{j}(w)+\frac{a_{j}(w)}{\pi} \int \frac{\vec{H}_{j} \bar{\partial} \varphi(z)}{a_{j}(z)(z-w)} d x d y \\
\vec{C}_{j}(w)=\vec{A}_{j}(w)-\vec{B}_{j}(w), \\
\vec{C}(w)=\sum_{j} \vec{C}_{j}(w) .
\end{gathered}
$$

The desired functions $\left\{\tilde{h}_{k}\right\}$ are just the components of the vector-valued function $\vec{h}-\vec{C}$. To see this, first note that

$$
\|(\vec{h}-\vec{C}) \cdot \vec{f}-g\|_{\infty} \leq\|\vec{h} \cdot \vec{f}-g\|_{\infty}+\|\vec{C} \cdot f\|_{\infty}
$$

The first term is small by our earlier remarks and if $z, w \in D\left(x_{j}, \delta\right)$ then the definition of $\delta$ gives

$$
\begin{aligned}
\left|\left(\vec{h}(z)-\vec{H}_{j}\right) \cdot \vec{f}(w)\right| & \leq|\vec{h}(z) \cdot \vec{f}(w)-g(w)|+\left|\vec{h}\left(z_{j}\right) \cdot \vec{f}(w)-g(w)\right| \\
& \leq 2 \varepsilon+2 \varepsilon .
\end{aligned}
$$


Thus the second term is bounded by

$$
\begin{aligned}
|\vec{C}(w) \cdot \vec{f}(w)| & \leq \sum_{j}\left\{\left|\frac{a_{j}(w)}{\pi} \int \frac{\left(\vec{h}(z)-\vec{H}_{j}\right) \cdot \vec{f}(w) \bar{\partial} \varphi(z)}{a_{j}(z)(z-w)} d x d y\right|\right. \\
& \left.+\left|\left(\vec{h}(w)-\vec{H}_{j}\right) \cdot \vec{f}(w)\right|\left|\varphi_{j}(w)\right|\right\} \\
& \leq \sum_{j} \frac{C \varepsilon}{\nu\left(1+\left|w-x_{j}\right|^{3}\right)} \int \frac{|\nabla \varphi(z)|}{|z-w|} d x d y+\varepsilon|\Phi(w)| \\
& \leq \varepsilon \sum_{j} \frac{C \delta}{\nu\left(1+\left|w-x_{j}\right|^{3}\right)}+\varepsilon|\Phi(w)| \\
& \leq C \varepsilon .
\end{aligned}
$$

It is easy to check that the components of $\vec{h}-\vec{C}$ are holomorphic on $\Omega$. Moreover, on the set $\{\Phi=1\}$,

$$
\vec{h}(w)-\vec{C}(w)=\sum_{j} \vec{H}_{j} \varphi_{j}(w)+\frac{a_{j}(w)}{\pi} \int \frac{\left(\vec{h}(z)-\vec{H}_{j}\right) \bar{\partial} \varphi(z)}{a_{j}(z)(w-z)} d x d y
$$

is a sum of continuous functions. Therefore the components of $\vec{h}-\vec{C}$ are continuous on $\bar{\Omega}$ and hence in $A(\Omega)$. This proves Theorem 1.4.

Next we will need the following lemma.

Lemma 6.1. If $\Omega$ is an open set which satisfies condition (1.1), then any $g \in$ $C(\bar{\Omega})$ can be uniformly approximated by a function $G \in C(\bar{\Omega})$ which is holomorphic where $g$ is and also on $\Omega \cap U, U$ a neighborhood of $\partial \Omega$.

The proof is exactly like the argument above. We fix $\varepsilon$ and choose $\delta$ so small that $|z-w|<\delta$ implies $|g(z)-g(w)|<\varepsilon$. Now choose $\left\{\varphi_{j}\right\}$ and $\left\{a_{j}\right\}$ exactly as above and set

$$
G(w)=g(w)-\sum_{j} \frac{a_{j}(w)}{\pi} \int \frac{(g(z)-g(w)) \bar{\partial} \varphi_{j}(z)}{a_{j}(z)(z-w)} d x d y .
$$

The arguments given above prove the lemma with $U=\left\{\sum \varphi_{j}=1\right\}$.

Corollary 6.2. If $\Omega$ is an open set and $g \in C(\bar{\Omega})$ then $g$ can be uniformly approximated by a continuous function on $\bar{\Omega}$ which is holomorphic on $\Omega \cap U$, $U$ a neighborhood of $\partial \Omega$.

We argue as above until we reach the definition of the $\left\{a_{j}\right\}$. Let $|E|$ denote the area of $E$. Recall that for a set $E,|E| \leq(\pi \alpha(E))^{2}$ (see [21]). If $\alpha\left(D\left(x_{j}, \delta / 4\right) \backslash \Omega\right)>\delta / 1000$ we define $a_{j}$ as above. Otherwise we have $\left|D\left(x_{j}, \delta / 4\right) \cap \Omega\right| \geq \delta^{2} / 100$ and so we can find a compact $E_{j} \subset D\left(x_{j}, \delta / 4\right) \cap \Omega$ such that $\alpha\left(E_{j}\right) \geq \delta / 100$. Now just take $a_{j} \in A_{E_{j}}$ with the desired estimates. Having defined $\left\{a_{j}\right\}$ for all $j$ we now write down $G$ as above and note that it 
has all the desired properties. In particular, it is holomorphic on $\Omega \cap U$ with $U=\left\{\sum \varphi_{j}=1\right\} \backslash \cup E_{j}$.

To prove Corollary 1.5 , let $\Omega=K^{\circ}$ and note that it is enough to approximate $g$ on $\bar{\Omega}$. Observe that since $g$ is continuous on $K$, we can apply Lemma 6.1 and so we may assume $g$ is holomorphic except on finitely many components of $K^{\circ}$. On these components we apply Theorem 1.1 to get an approximation. Thus we obtain a finite number of holomorphic functions $\left\{h_{k}\right\}$ which do the desired approximation on $K^{\circ}$. Corollary 1.5 now follows from Theorem 1.4. To prove Corollary 1.6 we need only observe that if $E$ contains an open connected subset of diameter $\geq r$ then $\alpha(E) \geq r$ (see [21]). Corollary 1.7 follows from a geometric characterization of the sets $K$ for which $A(K)$ is Dirichlet given in [20] (also see [7]). It says that $A(K)$ is Dirichlet iff each component of $K^{\circ}$ is simply connected and for all $x \in \partial K$ and all $r$ sufficently small

$$
\alpha\left(D(x, r) \backslash K^{\circ}\right) \geq r / 4 .
$$

This result does not actually require Theorem 1.1 since, as we shall see in $\S 9$, when $\Omega$ is simply connected Theorem 1.1 follows from the special case of the unit disk. It would be interesting to see if there was a direct proof of Corollary 1.7 from the definition of Dirichlet algebra.

The condition that

$$
\alpha(D(x, r) \cap E) \geq \varepsilon r
$$

for all $x \in E$ and $r$ small enough, is usually difficult to verify (see [21]), but the connected sets $E$ with this property have a nice geometrical characterization which can be checked in practice. If $E \subset \mathbb{C}$ is compact and $x \in E$ we say $x$ satisfies a double cone condition with respect to $E$ if there is a $\theta_{0} \in[0,2 \pi)$, $0<\varepsilon<\frac{\pi}{2}$ and $\delta>0$ such that

$$
\left\{x+r e^{i \theta}: 0<|r|<\delta,\left|\theta-\theta_{0}\right|<\frac{\pi}{2}-\varepsilon\right\} \cap E=\varnothing
$$

i.e., if there are two symmetric cones with vertex $x$ which do not hit $E$. Then if $E$ is connected, (6.1) holds if and only if the set of points in $E$ which satisfy a double cone condition has zero linear measure, i.e., it can be covered by a union of disks whose radii sum up to be as small as we wish [7, 8]. Using this criterion, it is easy to construct sets $K$ to which Corollary 1.5 applies. For example, one can easily obtain such $K$ which have arbitrarily small complementary components.

\section{Algebras Between $A(\Omega)$ AND $C(\partial \Omega)$}

In this section we will consider $A(\Omega)$ as a function algebra on $\partial \Omega$, and we will let $f$ denote both a function in $C(\partial \Omega)$ and its harmonic extension to $\Omega$ (which is continuous on $\bar{\Omega}$ since we are assuming $\Omega$ is regular). Thus we can say $f \in C(\partial \Omega)$ is holomorphic on some component of $\Omega$ or refer to the value $f(z)$ for some $z \in \Omega$ without introducing more notation. When $\Omega$ is the unit disk, John Wermer [51] classified all the closed subalgebras of $C(\partial \Omega)$ which 
contain $A(\Omega)$ : there are none except $C(\partial \Omega)$ and $A(\Omega)$. In this section we will describe all these subalgebras in the case when Theorems 1.1 and 1.4 apply to $\Omega$, i.e., when $\Omega$ satisfies (1.1) and all its components are Widom domains. Other situations have been considered in papers such as [5, 13 and 26].

Suppose $\left\{\Omega_{j}\right\}$ are the components of $\Omega$ and let $U$ denote the union of some subcollection of the $\left\{\Omega_{j}\right\}$. Now define

$$
A_{U}=\{f \in C(\partial \Omega): f \text { is holomorphic on } U\} .
$$

Then $A_{U}$ is clearly a closed subalgebra of $C(\partial \Omega)$ which contains $A(\Omega)$. We shall prove these are the only such algebras.

Corollary 7.1. Suppose $\Omega$ is an open set which satisfies (1.1) and such that each component is a Widom domain. Then the only closed subalgebras of $C(\partial \Omega)$ containing $A(\Omega)$ are of the form $A_{U}$. Moreover, distinct $U$ 's give rise to distinct $A(U)$ 's. In particular, the maximal subalgebras of $C(\partial \Omega)$ containing $A(\Omega)$ are exactly the algebras $A_{\Omega_{j}}$.

Let $A$ be a closed subalgebra of $C(\partial \Omega)$ which contains $A(\Omega)$. Let $U$ be the largest subset of $\Omega$ such that every element of $A$ is holomorphic on $U$, i.e., $A \subset A_{U}$, and $A_{U}$ is the smallest such algebra. We will show $A_{U}=A$. Since the algebras $A_{U}$ are clearly distinct for distinct $U$ 's this proves the lemma (if $U_{1} \neq U_{2}$ consider $f(z)=1 /(z-a)$ for an $\left.a \in U_{1} \backslash U_{2}\right)$.

Take $g \in A_{U}$. We will show $g \in A$. By Lemma 6.1 we may assume $g$ is also holomorphic on a neighborhood of $\partial \Omega$. Thus it fails to be holomorphic on only a finite number of components $\left\{\Omega_{j}\right\}, j=1, \ldots, N$. For each $j, A$ contains a function $f_{j}$ which is not holomorphic on $\Omega_{j}$ (by the definition of $U$ and the convention introduced above). We claim that

$$
f(z)=\sum_{j} c_{j} f_{j}(z)
$$

is not holomorphic on any $\Omega_{j}$ for some choice of constants $\left\{c_{j}\right\}$. To see this, note that if $h_{1}$ and $h_{2}$ are harmonic functions on a connected set, and $h_{2}$ is not holomorphic, then $h_{1}+t h_{2}$ can be holomorphic for at most one value of $t$. So using induction, we can easily construct the desired $\left\{c_{j}\right\}$. Thus by Theorem 1.1 we can find functions $\left\{h_{j k}\right\}$ in $H^{\infty}\left(\Omega_{j}\right)$ such that $\sum h_{j k} f^{k}$ uniformly approximates $g$ on $\Omega_{j}$. This, plus the fact that $g$ is already holomorphic on the rest of $\Omega$, implies there are functions $\left\{h_{k}\right\}$ in $H^{\infty}(\Omega)$ such that $\sum h_{k} f^{k}$ approximates $g$ on all on $\Omega$. Applying Theorem 1.4 we may therefore assume $\left\{h_{k}\right\} \subset A(\Omega)$. Since $f \in A$ we have $g \in A(\Omega)[f] \subset A$ as required. This completes the proof of Corollary 7.1.

Of course, if $K$ is a compact set which satisfies the hypotheses of Corollary 1.5 , then Corollary 7.1 applies to $K^{o}$, so we obtain a description of all the closed algebras between $A(K)$ and $C(\partial K)$. Better results are possible. For example, in [5] it is stated that if $K^{o}=\Omega$ is connected and $K \cap \bar{D}(x, r)$ does 
not separate the plane for some disk centered on $\partial \Omega$, then $A(K)$ is maximal in $C(\partial K)$.

In [26], Hoffman and Singer consider the algebra $A(\Omega)$ when $E=\overline{\mathbb{C}} \backslash \Omega$ consists of finitely many arcs which locally have positive area and they show that $A(\Omega)$ is contained in a maximal proper subalgebra of $C(E)$, though they do not show $A(\Omega)$ itself is maximal. If we replace their hypothesis with the hypothesis that $E$ satisfies (6.1), then Corollary 7.1 implies $A(\Omega)$ is maximal in $C(E)$. Since $(6.1)$ is implied by the condition

$$
|D(x, r) \cap E| \geq \varepsilon r^{2}
$$

we see we have obtained a slightly stronger conclusion from a slightly stronger hypothesis.

It would be very interesting to understand what happens when we drop our (rather restrictive) hypotheses on $\Omega$. In particular, how important is the assumption that the components are Widom domains? My first impression is that the result should be true without this assumption. However, some condition on the "thickness" of the boundary is needed. As an extreme example, consider $\Omega=\Omega_{1} \cup \Omega_{2}=\overline{\mathbb{C}} \backslash \mathbf{T}$. Then $A(\Omega)$ is trivial, and $C(\mathbf{T})$ certainly contains many closed subalgebras other than $A\left(\Omega_{1}\right)$ and $A\left(\Omega_{2}\right)$, e.g., the functions which vanish on a closed proper subset $E \subset \mathbf{T}$.

What happens if $\alpha(D(x, r) \backslash \Omega)$ is positive for every disk centered on $\partial \Omega$, but (1.1) fails? One can easily construct an explicit example where this happens as follows. Let $\Gamma$ be an arc connecting 0 to 1 which satisfies condition (6.1) and remains in the region $\{x, y:|y| \leq|x(x-1)|\}$. Now let $E \subset[0,1]$ be a Cantor set of positive length and replace the complementary intervals by appropriately scaled copies of $\Gamma$. Then we obtain an arc which has positive continuous analytic capacity in every neighborhood of every point, but such that (6.1) fails. If $\Omega$ is the complement of this arc, is $A(\Omega)$ maximal in $C(\partial \Omega)$ ?

\section{The Chang-Marshall theorem}

We will now relate our earlier ideas to the well-known theorem of Chang [12] and Marshall [33] (also see [23, Chapter IX]). In this section $H^{\infty}=H^{\infty}(D)$ will be considered both as a function algebra on $D$ and on $\mathbf{T}$.

Theorem 8.1 (Chang-Marshall). Suppose $H^{\infty}(\mathbf{T}) \subset A \subset L^{\infty}(\mathbf{T})$ is a closed algebra. Then $A$ is generated by $H^{\infty}$ and the complex conjugates of a collection of inner functions. (In fact, this collection can be taken to consist of interpolating Blaschke products.)

The original proof is nonconstructive, but Sundberg [46] and Vol'berg [50], have shown how to make it constructive. We shall show below how to replace a remaining duality argument by an explicit construction. In the proof of this theorem one can easily reduce to the case $A=H^{\infty}[f],|f|=1$ on $\mathbf{T}$ and $\bar{f} \in A$ (see [ 46 or 22]). We then fix $\varepsilon>0$ and construct a certain interpolating Blaschke product $B$ (depending on $\varepsilon$ ). First one shows $\bar{B} \in A$. In the original 
proof this is done by an argument involving the maximal ideal space of $H^{\infty}$, and so is nonconstructive. However, Sundberg [46] showed $\bar{B} \in A$ by an explicit construction and an even simpler proof was later given by Vol'berg [50]. The second step is to prove

$$
\operatorname{dist}\left(f, H^{\infty}(\mathbf{T})[\bar{B}]\right) \leq \varepsilon
$$

(so letting $\varepsilon \rightarrow 0$ gives a collection of Blaschke products which generate $A=$ $\left.H^{\infty}[f]\right)$. Originally, this was proved using duality, i.e., showing

$$
\operatorname{dist}\left(f, H^{\infty}(\mathbf{T})[\bar{B}]\right)=\inf _{n} \sup _{F \in H_{0}^{1},\|F\|=1} \frac{1}{2 \pi} \int f B^{n} F d \theta,
$$

and then estimating the right-hand side using Littlewood-Paley type arguments. We shall prove it by explicitly approximating $f$ by elements of $H^{\infty}[\bar{B}]$. We shall need the following

Lemma 8.2. Suppose $f$ is a bounded harmonic function on $D$ which is unimodular on $\mathbf{T}$ and let $\varepsilon>0$ be given. Then there exists $\delta, \nu>0$, an open set $\Omega \subset D$ with boundary $\Gamma, a C^{\infty}$ function $F$ on $D$ and a Blaschke product $B$ with zeros $\left\{z_{n}\right\}$ on $\Gamma$ such that

(1) For a.e. $z \in \mathbf{T}, r z \in \Omega$ for all $r$ close enough to 1 .

(2) Arclength on $\Gamma$ is a Carleson measure.

(3) $|f(z)| \leq 1-\nu$ for $z \in \Gamma$.

(4) $|B(z)| \leq 1 / 2$ for $z \in \Gamma$.

(5) $\bar{\partial} F$ is supported in $\{z: \operatorname{dist}(z, \Gamma) \leq \delta(1-|z|)\}$ and satisfies

$$
|\bar{\partial} F(z)| \leq C /(\delta(1-|z|)) .
$$

(6) $|f(z)-F(z)| \leq \varepsilon$ for $z \in \Omega$.

The first four conditions are just Marshall's original construction (see [33 or 22, Theorem VIII.4.1]), and (5) and (6) are just a minor variant. Using his arguments one shows $f$ can be approximated by a holomorphic function $F$ on $\Omega$. Setting $F=0$ off $\Omega$ and "smoothing" gives the rest of Lemma 8.2.

For completeness, we begin with a sketch of Vol'berg's proof that $\bar{B} \in$ $H^{\infty}[f]$. Let $f$ and $B$ be as above and fix $\eta>0$. We shall prove

$$
\operatorname{dist}\left(\bar{B}, H^{\infty}(\mathbf{T})[f]\right)<C \eta \text {. }
$$

Since $|B|$ has radial limit 1 a.e. on $\mathbf{T}$, it suffices to approximate $\bar{B}$ on a set of the form $S_{\eta}(B)=\{z:|B(z)|>1-\eta\}$. Fix $\lambda \in \mathbf{T}$ and define

$$
a_{\lambda}(z)=\operatorname{Re}(1-\bar{\lambda} f(z)), \quad A_{\lambda}(z)=\exp \left(-a_{\lambda}(z)-i a_{\lambda}^{*}(z)\right) .
$$

Then $\left|A_{\lambda}\right|$ is bounded by 1 and is near 1 only when $f$ is near $\lambda$. We are assuming that $\left|f\left(z_{n}\right)\right|<1-\nu$ so $\left|A_{\lambda}\left(z_{n}\right)\right|<\exp (-\nu)<1$. Thus by replacing $A_{\lambda}$ by a large power of itself we may assume $A_{\lambda}\left(z_{n}\right)<\eta$. Since $\left\{z_{n}\right\}$ is an interpolating sequence we can find holomorphic Pehr Beurling functions $\left\{h_{n}\right\}$ such that

$$
h_{n}\left(z_{n}\right)=1 \text {, }
$$




$$
\begin{gathered}
h_{n}\left(z_{m}\right)=0, \quad n \neq m, \\
\sum_{n}\left|h_{n}(z)\right| \leq C, \quad z \in D .
\end{gathered}
$$

For a proof see [22, Theorem VII.2.1] (using duality) or [30, Theorem 6] (explicit construction). Now set

$$
\begin{gathered}
G_{\lambda}(z)=\frac{A_{\lambda}(z)-\sum_{n} h_{n}(z) A_{\lambda}\left(z_{n}\right)}{B(z)}, \\
E_{\lambda}=\left\{z \in D:\left|A_{\lambda}(z)-1\right|<\eta\right\} .
\end{gathered}
$$

Then $G_{\lambda} \in H^{\infty}$ and for $z \in E_{\lambda}$,

$$
\left|G_{\lambda}(z) B(z)-1\right|<C \eta .
$$

Thus if $z \in S_{\eta}(B) \cap E_{\lambda}$,

$$
\left|G_{\lambda}(z)-\bar{B}(z)\right| \leq C \eta .
$$

Thus we have approximated $\bar{B}$ on pieces of $S_{\eta}(B)$ and all that is left is to "glue together" these approximations. As in $\S \S 2$ and 3 , let $\left\{P_{j}(x, y)\right\}$ be a partition of unity on $\{1-\eta / 4 \leq|z| \leq 1\}$ with $\operatorname{supp}\left(P_{j}\right) \subset D\left(\lambda_{j}, \eta / 2\right),\left\{\lambda_{j}\right\} \subset \mathbf{T}$. Then

$$
G(z)=\sum_{j} G_{\lambda_{j}}(z) P_{j}(\operatorname{Re}(f), \operatorname{Im}(f))
$$

is in $H^{\infty}[f]$ since $\operatorname{Re}(f)$ and $\operatorname{Im}(f)$ are and since the $\left\{P_{j}\right\}$ can be approximated by polynomials. Finally, if $z \in S_{\eta}(B) \cap E_{\lambda}$,

$$
|G(z)-\bar{B}(z)| \leq C \sup _{j: f(z) \in \operatorname{supp}\left(P_{j}\right)}\left|G_{\lambda_{j}}(z)-\bar{B}(z)\right| \leq C \eta
$$

as required. This completes Vol'berg's construction.

Now we turn to approximating $f$ using $\bar{B}$. Take $\Omega, F$ and $B$ as in Lemma 2. Fix $\lambda$ with $3 / 4 \leq|\lambda| \leq 1$. We wish to solve the equation

$$
\bar{\partial} H_{\lambda}=\frac{\bar{\partial} F}{B-\lambda}
$$

with an $L^{\infty}$ estimate on $H_{\lambda}$ which is independent of $\lambda$. We can do this because of conclusions (4) and (6) of the lemma. More precisely, because of (4) we can write $\operatorname{supp}(\bar{\partial} F)=\cup D_{j}$ where $\left\{D_{j}\right\}$ is a disjoint collection of sets of hyperbolic diameter $\delta$ and we can find holomorphic functions $\left\{h_{j}\right\}$ which satisfy

$$
\begin{gathered}
\left|h_{j}(z)\right|>\frac{1}{2}, \quad z \in D_{j}, \\
\sum_{j}\left|h_{j}\right| \leq C .
\end{gathered}
$$

(For details see $\S 3$ or [22, Chapter VIII].) As in $\S 3$ we set

$$
H_{\lambda}(z)=\sum_{j} \frac{h_{j}(z)}{\pi} \int_{D_{j}} \frac{\bar{\partial} F(w) d x d y}{h_{j}(w)(B(w)-\lambda)(z-w)} .
$$


By conditions (4) and (6) and Schwarz's lemma, $\bar{\partial} F(w) \neq 0$ imples $|B(w)| \leq$ $1 / 2+\delta<2 / 3$, so for such a $w,|B(w)-\lambda| \geq 1 / 12$. Thus the series defining $H_{\lambda}$ converges absolutely and with a bound $C_{1}$ independent of $\lambda$. Thus

$$
G_{\lambda}=F-(B-\lambda) H_{\lambda}
$$

is a bounded holomorphic function on $D$ which approximates $F$ near the set $\{B=\lambda\}$. Now take a partition of unity $\left\{P_{j}(x, y)\right\}$ on $\{3 / 4 \leq|z| \leq 1\}$ with $\operatorname{supp}\left(P_{j}\right) \subset D\left(\lambda_{j}, \varepsilon / C_{1}\right)$. Then

$$
G(z)=\sum_{j} G_{\lambda_{j}}(z) P_{j}(\operatorname{Re}(B), \operatorname{Im}(B))
$$

is in $H^{\infty}[\bar{B}]$ (just as above) and for $z \in \Omega \cap S_{1 / 4}(B)$

$$
\begin{aligned}
|f(z)-G(z)| & \leq|f(z)-F(z)|+|F(z)-G(z)| \\
& \leq \varepsilon+\sum_{j: B(z) \in \operatorname{supp}\left(P_{j}\right)}\left|H_{\lambda}(z)\right|\left|B(z)-\lambda_{j}\right| \\
& \leq \varepsilon+10 C_{1} \varepsilon / C_{1} \\
& \leq 11 \varepsilon .
\end{aligned}
$$

Since the characteristic function of $\Omega \cap S_{1 / 4}(B)$ has radial limit 1 a.e. on $\mathbf{T}$, we deduce that $|f(z)-G(z)| \leq 11 \varepsilon$ a.e. on $\mathrm{T}$. This completes the proof of the Chang-Marshall theorem.

\section{SPECial Cases of Theorem 1.1}

We can give simpler proofs of Theorem 1.1 in various special cases. We have already discussed one simplification when $\Omega$ is finitely connected. If $\Omega$ is simply connected then Theorem 1.1 follows from the special case of the unit disk. To see this, let $f$ and $g$ be as before. We can approximate $g$ by a sum of functions $g_{1}+g_{2}$ where $g_{1}$ has compact support in $\Omega$ and $g_{2}$ is harmonic on $\Omega$ and continuous on $\bar{\Omega}$. Let $\Phi$ denote a conformal mapping from the unit disk to $\Omega$. Let $f, g_{1}, g_{2}$ also denote the pullbacks of these functions to $D$ via $\Phi$. Then clearly $g_{1} \in H^{\infty}(D)[f]$ since $g_{1}$ is still continuous and we are assuming Theorem 1.1 for the disk. The function $g_{2}$ is not necessarily continuous, but it is in VMO [7, Lemma 5.1], so we can write $g_{2}=u+v$ where $u$ and $v$ are harmonic, $u$ is continuous and $v$ is in BMO with as small a norm as we wish. Therefore, by a theorem of Varopolos [47, 48], we can find a function $\tilde{v}$ on $D$ which agrees with $v$ on $\mathbf{T}$ and such that $|\nabla \tilde{v}|$ is a Carleson measure with small norm. Therefore we can solve the $\bar{\partial}$ problem $\bar{\partial} b=\bar{\partial} \tilde{v}$ with a function $b$ whose $L^{\infty}(\mathbb{T})$ norm is small. Since $u$ is continuous, it is in $H^{\infty}(D)[f]$. Because $\tilde{v}-b$ is bounded and holomorphic and

$$
\left\|g_{1}-u-(\tilde{v}-b)\right\|=\|v-(\tilde{v}-b)\| \leq \sup _{\mathbf{T}}|(v-\tilde{v})+b|=\sup _{\mathbf{T}}|b|
$$

is as small as we wish, we see that $g_{2}$ is also in $H^{\infty}(D)[f]$, as required. 
Another simplification is possible if we assume $f$ is real-valued in Theorem 1.1. In that case $f$ has a real valued conjugate $f^{*}$ (after pulling back to $D$ ), so $h=f+i f^{*}$ is holomorphic. Thus $e^{h}$ is bounded and holomorphic, and if we assume $\|f\|_{\infty} \leq 1$ then

$$
\left|e^{h(z)}-e^{\lambda}\right| \sim \operatorname{dist}(h(z), \lambda+\mathscr{L}) .
$$

Thus in Lemma 3.1 we can just take $A$ to be this function, avoiding the argument in $\S 5$.

If $f=u+i v$ has a single-valued conjugate on $\Omega$, then $h=\left(u-v^{*}\right)-i\left(v+u^{*}\right)$ is holomorphic on $\Omega$. Let $w=\log (h-\lambda)-2 H$ (where $H$ denotes the harmonic majorant of $\left.\log ^{+}|h-\lambda|\right)$. Because $\Omega$ is a Widom domain, we can use Widom's theorem to find a bounded function $g$ such that

$$
A_{\lambda}=g e^{w+i w^{*}}
$$

is a bounded, single-valued, holomorphic function (on the unit disk this would just follow from the canonical factorization theorem [22, Section II.5]). Then one can easily check that

$$
\left|A_{\lambda}(z)\right| \leq C \min \left(|h(z)-\lambda|, \frac{1}{|h(z)-\lambda|}\right) .
$$

We can use this to set up a $\bar{\partial}$ problem (as in $\S 2$ ) and then approximate the desired function on sets of the form

$$
F_{\lambda}=\{h=\lambda\} \cup\{|h-\lambda| \geq M\}
$$

and thus on small neighborhoods of such sets. Covering the $\lambda$-plane with finitely many such neighborhoods and constructing the appropriate partitions of unity gives the result. This construction is essentially the proof given by Axler and Shields [3] on the unit disk, although they phrase it in the language of functional analysis. In this argument we again used the assumption than $\Omega$ is Widom, but in a much less essential way that before. Here we only needed to find a function corresponding to one particular character, whereas our hypothesis implies we could find functions corresponding to any character.

If we assume that $f$ is real valued and continuous on $\bar{\Omega}$ then the proof becomes quite easy in many cases. We take the unit disk as an example. Assume the range of $f$ is the interval $[-1,1]$. For each $\lambda \in[-1,1]$ the level set $E_{\lambda}=\{f=\lambda\}$ is a compact subset of the closed disk with no interior and which does not separate the plane (otherwise $f$ would be constant!). Thus we can approximate $g$ on $E_{\lambda}$ by a function $g_{\lambda}$ in $A(D)$ (in fact, by a polynomial using Lavrentiev's theorem) and this approximation remains good on $E_{\sigma}$ for all $\sigma$ in a small open interval around $\lambda$. These intervals cover $[-1,1]$ so we can choose a finite subcover $\left\{I_{j}\right\}, j=1, \ldots, N$, and denote the corresponding $g_{\lambda}$ 's by $\left\{g_{j}\right\}$. Now choose a partition of unity $\left\{P_{j}\right\}$ of polynomials on $[-1,1]$ such that

$$
\sup \left\{\left|P_{j}(f(z))\right|\left|g_{j}(z)\right|: f(z) \notin I_{j}\right\} \leq \varepsilon / N .
$$


Then

$$
Q(x)=\sum_{j} P_{j}(f(z)) g_{j}(z)
$$

does the required approximation. Related arguments are given in [3, 26, 40]. This proof shows $A(K)[f]=C(K)$ whenever we know that $A(K)$ is dense in $C(E), E$ a level set of $f$. Alexander Izzo [28] has proven this whenever $E$ is a subset of $K$ with no interior and such that each component of the complement of $E$ contains a component of the complement of $K$. Thus he deduces that for any compact $K, A(K)[f]=C(K)$ whenever $f$ is a real-valued, continuous function on $K$ which is harmonic on $K^{\circ}$, but not constant on any component of $K^{\circ}$. In fact, since we never use the harmonicity of $f$ except to describe the geometry of its level sets, the proof shows $A(K)[f]=C(K)$ whenever $f$ is a continuous, real-valued function such that $\{f=\lambda\}$ satisfies the hypothesis of Izzo's theorem for each $\lambda$. These conditions are also necessary, so he obtains a characterization of all continuous, real-valued functions $f$ on $K$ such that $A(K)[f]=C(K)$. On the unit disk this was observed by Mergelyan [34].

\section{FinAl REMARKS}

Theorem 1.1 can probably be generalized, but I do not think the technique used in this paper can be greatly extended, so we need another approach. In particular, it would be interesting to see a regular, non-Widom, domain for which Theorem 1.1 is true. For example, if $\Omega$ is the complement of a "square" Cantor set then it is regular but not Widom. Does Theorem 1.1 hold for such a domain? Part of the problem here may be to determine which characters of $\Gamma$ can actually occur as the argument of a multi-valued holomorphic function on $\Omega$. Another special case to consider is the class of Denjoy domains, i.e., $\partial \Omega=E \subset \mathbf{R}$ (see $[11,31,23]) . E$ is called homogeneous if there is an $\varepsilon>0$ such that

$$
|(x-r, x+r) \cap E| \geq \varepsilon r
$$

for all $r>0$ and every $x \in E$, and in this case the projections discussed in $\S 4$ exist. For what other Denjoy domains is Theorem 1.1 true?

We can also consider extending Corollary 1.5. Since if $f$ is real valued it holds for every compact $K$, one might expect it to hold for complex-valued functions in the general case. If not, it should interesting to understand why it fails.

Does Theorem 1.1 hold on general domains if we assume $f$ has a singlevalued harmonic conjugate? If it has a single-valued, bounded harmonic conjugate, we proved in $\S 2$ that this is true. This probably fails, but if it were true one might be able to "interpolate" between this result and Theorem 1.1 to obtain classes of harmonic functions on a given domain such that $C(\bar{\Omega}) \subset H^{\infty}(\Omega)[f]$ in terms of the periods of $f^{*}$. However, it may be that these periods are irrelevant to the problem and that their apparent importance may simply be an artifact of our techniques. 
Finally, can one combine the ideas of this paper with the several complex variables approach discussed in the introduction, or at least understand the relationship between them? In particular, how important is the smoothness at the boundary? For example, Corollary 1.5 shows $C(\bar{D})=A(D)[f]$ if $f$ is harmonic, continuous and not holomorphic. Can it be deduced from the smooth case? Is there a proof which does not use Theorem 1.1? If not, does Theorem 1.1 on $D$ somehow follow from the special case of continuous functions?

\section{REFERENCES}

1. L. Ahlfors, Conformal invariants: topics in geometric function theory, McGraw-Hill, New York, 1973.

2. S. Axler and P. Gorkin, Algebras on the disk and doubly commuting multiplication operators, Trans. Amer. Math. Soc. 309 (1988), 711-723.

3. S. Axler and A. Shields, Algebras generated by analytic and harmonic functions, Indiana Univ. Math. J. 36 (1987), 631-638.

4. A. Baernstein, Analytic functions with bounded mean oscillation, Aspects of Contemporary Complex Analysis, Academic Press, New York, 1980, pp. 3-36.

5. B. T. Batikyan and S. A. Grigoryan, On uniform algebras containing $A(K)$, Uspekhi Mat. Nauk 40 (1985), 167-208; Russian Math. Surveys 40 (1985), 205-206.

6. A. Beardon, The geometry of discrete groups, Graduate Texts in Math. 91, Springer-Verlag, Berlin, 1983.

7. C. J. Bishop, Constructing continuous functions holomorphic off a curve, J. Funct. Anal. (to appear)

8. C. J. Bishop, L. Carleson, J. B. Garnett and P. W. Jones, Harmonic measures supported on curves, Pacific J. Math. (to appear)

9. A. Browder and J. Wermer, Some algebras of functions on an arc, J. Math. Mech. 12 (1963), 119-130.

10. L. Carleson, Interpolation by bounded analytic functions and the corona problem, Ann. of Math. 76 (1965), 547-559.

11. of Antoni Zygmund, Vol. II, edited by W. Beckner et al., Wadsworth, Belmont, Calif., 1983, pp. 349-372.

12. S.-Y. A. Chang, A characterization of Douglas subalgebras, Acta Math. 137 (1976), 81-89.

13. E. M. Čirka, Approximation by holomorphic functions on smooth manifolds in $\mathbf{C}^{n}$, Mat. Sb. 78 (1969), 101-123=Math. USSR-Sb. 7 (1969), 95-114.

14. A. M. Davie, T. W. Gamelin and J. B. Garnett, Distance estimates and pointwise bounded density, Trans. Amer. Math. Soc. 175 (1973), 37-68.

15. C. J. Earle and A. Marden, On Poincaré series with applications to $H^{p}$ spaces on bordered Riemann surfaces, Illinois J. Math. 13 (1969), 202-219.

16. F. Forelli, Bounded holomorphic functions and projections, Illinois J. Math. 10 (1966), 367380.

17. H. Furstenberg, Recurrence in ergodic theory and combinatorial number theory, Princeton Univ. Press, Princeton, N. J., 1981.

18. T. W. Gamelin, Uniform algebras, Prentice-Hall, Englewood Cliffs, N. J., 1969.

19. T. W. Gamelin and J. B. Garnett, Constructive techniques in rational approximation, Trans. Amer. Math. Soc. 143 (1969), 187-200.

20. __ Pointwise bounded approximation and Dirichlet algebras, J. Funct. Anal. 8 (1971), 360404. 
21. J. B. Garnett, Analytic capacity and measure, Lecture Notes in Math., vol. 297, SpringerVerlag, Berlin, 1972.

22. __ Bounded analytic functions, Academic Press, New York, 1981.

23. J. B. Garnett and P. W. Jones, The corona theorem for Denjoy domains, Acta Math. 155 (1985), 17-40.

24. W. K. Hayman and Ch. Pommerenke, On analytic functions of bounded mean oscillation, Bull. London Math. Soc. 10 (1978), 219-224.

25. K. Hoffman, Banach spaces of analytic functions, Prentice-Hall, Englewood Cliffs, N. J., 1962.

26. K. Hoffman and I. M. Singer, Maximal algebras of continuous functions, Acta Math. 103 (1960), 217-241.

27. L. Hørmander and J. Wermer, Uniform approximation on compact sets in $\mathbb{C}^{n}$, Math. Scand. 23 (1968), 5-21.

28. A. Izzo, Thesis, Univ. of California at Berkeley, 1989.

29. P. W. Jones, Carleson measures and the Fefferman-Stein decomposition of $B M O(\mathbf{R})$, Ann. of Math. 111 (1980), 197-208.

30. P. W. Jones, $L^{\infty}$ estimates for the $\bar{\partial}$ problem in a half-plane, Acta Math. 150 (1983), $137-$ 152.

31. P. W. Jones and D. Marshall, Critical points of Green's function, harmonic measure, and the corona problem, Ark. Mat. 23 (1985), 281-314.

32. L. Keen, Canonical polygons for finitely generated Fuchsian groups, Acta Math. 115 (1966), 1-16.

32. D. E. Marshall, Subalgebras of $L^{\infty}$ containing $H^{\infty}$, Acta Math. 137 (1976), 91-98.

34. S. N. Mergelyan, On a theorem of M. A. Lavren'ev, Dokl. Akad. Nauk SSSR 77 (1951), 565568; English transl., Amer. Math. Soc. Transl. (1) 3 (1962), 281-286.

35. __ On the representation of functions by series of polynomials on closed sets, Dokl. Akad. Nauk SSSR 78 (1951), 405-408; English transl., Amer. Math. Soc. Transl. (1) 3 (1962), 287293.

36. A. G. O'Farrell and K. J. Preskenis, Approximation by polynomials in two complex variables, Math. Ann. 246 (1980), 225-232.

37. __ Approximation by polynomials in two diffeomorphisms, Bull. Amer. Math. Soc. 10 (1984), 105-107.

38. R. F. Olin, Functional relationships between a subnormal operator and its minimal normal extension, Pacific J. Math. 63 (1976), 221-229.

39. Ch. Pommerenke, On the Green's function of Fuchsian groups, Ann. Acad. Sci. Fenn. 2 (1976), 409-427.

40. K. J. Preskenis, Approximation on disks, Trans. Amer. Math. Soc. 171 (1972), 445-467.

41. _ Another view of the Weierstrass theorem, Proc. Amer. Math. Soc. 54 (1976), 109-113.

42. W. Rudin, Spaces of type $H^{\infty}+C$, Ann. Inst. Fourier (Grenoble) 25 (1975), 99-125.

43. D. Sarason, Algebras of functions of the circle, Bull. Amer. Math. Soc. 79 (1973), 286-299.

44. D. Stegenga, A geometric condition which implies BMOA, Proc. Sympos. Pure Math., vol. 35, Amer. Math. Soc., Providence, R. I., 1979, pp. 427-430.

45. D. Stegenga and K. Stephenson, A geometric characterization of analytic functions with bounded mean oscillation, J. London Math. Soc. 24 (1981), 243-254.

46. C. Sundberg, A constructive proof of the Chang-Marshall theorem, J. Funct. Anal. 46 (1982), 239-245.

47. N. Th. Varopoulos, BMO functions and the $\bar{\partial}$ equation, Pacific J. Math. 71 (1977), 221-273.

48. __ A remark on BMO and bounded harmonic functions, Pacific J. Math. 73 (1977), 257-259.

49. A. G. Vitushkin, Analytic capacity of sets and problems in approximation theory, Uspekhi Mat. Nauk 22 (1967), 141-199=Russian Math. Surveys 22 (1967), 139-199. 
50. A. L. Vol'berg, A constructive proof of the Chang-Marshall theorem, (Russian, English summary), Zap. Nauchn. Sem. Leningrad. (LOMI) 141 (1985), 149-153.

51. J. Wermer, On algebras of continuous functions, Proc. Amer. Math. Soc. 4 (1953), 866-869.

52. __ Approximations on a disk, Math. Ann. 155 (1964), 331-333.

53. _ _ Polynomially convex disks, Math. Ann. 158 (1965), 6-10.

54. __ Banach algebras and several complex variables, Graduate Texts in Math. 35, SpringerVerlag, Berlin, 1976.

55. H. Widom, The maximum principle of multi-valued analytic functions, Acta Math. 126 (1971), 63-82.

Department of Mathematics, University of California, Los Angeles, California 90024 